\title{
String bracket and flat connections
}

\author{
HOSSEIN ABBASPOUR \\ MAHMOUD ZEINALIAN
}

Let $G \rightarrow P \rightarrow M$ be a flat principal bundle over a compact and oriented manifold $M$ of dimension $m=2 d$. We construct a map $\Psi: H_{2 *}^{S^{1}}(L M) \rightarrow \mathcal{O}(\mathcal{M C})$ of Lie algebras, where $H_{2 *}^{S^{1}}(L M)$ is the even dimensional part of the equivariant homology of $L M$, the free loop space of $M$, and $\mathcal{M C}$ is the Maurer-Cartan moduli space of the graded differential Lie algebra $\Omega^{*}(M$, ad $P)$, the differential forms with values in the associated adjoint bundle of $P$. For a 2-dimensional manifold $M$, our Lie algebra map reduces to that constructed by Goldman [17]. We treat different Lie algebra structures on $H_{2 *}^{S^{1}}(L M)$ depending on the choice of the linear reductive Lie group $G$ in our discussion. This paper provides a mathematician-friendly formulation and proof of the main result of Cattaneo, Frohlich and Pedrini [3] for $G=\operatorname{GL}(n, \mathbb{C})$ and $\operatorname{GL}(n, \mathbb{R})$ together with its natural generalization to other reductive Lie groups.

55P35; 57R19, 58A10

\section{Introduction}

The precursors to the Chas-Sullivan string bracket [5] were Goldman's Lie algebra structures [17] on certain vector spaces based on the homotopy classes of closed curves on a closed orientable surface $S$. The simplest one of these was defined on the vector space $\mathbb{R} \hat{\pi}$ generated by the set $\hat{\pi}$ of free homotopy classes of closed oriented curves on $S$. There was a similar construction of a Lie algebra based on unoriented curves. For the moment, let us talk about $\mathbb{R} \hat{\pi}$ for which the bracket of two equivalence classes of curves is a signed summation of the curves obtained by breaking and reconnecting two transversal representatives at each of their intersection points one at a time. The relevance, and more importantly the universality, of this algebraic object to geometry was established by defining a map $\gamma \mapsto f_{\gamma}$, from $\mathbb{R} \hat{\pi}$ to the Poisson algebra of smooth functions on the symplectic space $\operatorname{Hom}(\pi, G) / G$ of representations of $\pi$ into $G=\operatorname{GL}(n, \mathbb{C})$ or $\operatorname{GL}(n, \mathbb{R})$. Said slightly differently, to a free homotopy class of a closed curve one assigns a function on the moduli space of all flat connections modulo the gauge group. The value of the function $f_{\gamma}$ at an equivalence class $\alpha$ is the trace of the holonomy with respect to the flat connection representing $\alpha$ along a oriented 
closed curve representing the free homotopy class $\gamma$. The Poisson bracket of two such functions is identified as (see [17])

$$
\left\{f_{\gamma}, f_{\lambda}\right\}=\sum_{p \in \gamma \# \lambda} \varepsilon(p ; \gamma, \lambda) f_{\gamma_{p} \lambda_{p}},
$$

where $\gamma_{p} \lambda_{p}$ denotes the product of the elements $\gamma_{p}, \lambda_{p} \in \pi_{1}(S ; p)$, and $\varepsilon(p ; \gamma, \lambda)=$ \pm 1 denotes the oriented intersection number of $\gamma$ and $\lambda$ at $p$.

Goldman [17] showed that

$$
[\gamma, \lambda]=\sum_{p \in \gamma \# \lambda} \varepsilon(p ; \gamma, \lambda) \gamma_{p} \lambda_{p}
$$

defines a Lie bracket on $\mathbb{R} \hat{\pi}$, the free vector space generated by the conjugacy classes of $\pi$. In particular this means that the map $\gamma \mapsto f_{\gamma}$ is a map of Lie algebras.

Similarly, he showed that the Lie algebra structure on the vector space based on the set of free homotopy classes of unoriented curves corresponds to the case where $G$ is $\mathrm{O}(p, q), \mathrm{O}(n, \mathbb{C}), \mathrm{U}(p, q), \mathrm{Sp}(n, \mathbb{R})$, or $\mathrm{Sp}(p, q)$. In this case the Poisson bracket has the formula

$$
\left\{f_{\gamma}, f_{\lambda}\right\}=\frac{1}{2} \sum_{p \in \gamma \# \lambda} \varepsilon(p ; \gamma, \lambda)\left(f_{\gamma_{p} \lambda_{p}}-f_{\gamma_{p} \lambda_{p}^{-1}}\right),
$$

where once again $\gamma_{p} \lambda_{p}$ and $\gamma_{p} \lambda_{p}^{-1}$ denote the product of $\gamma_{p}$ with $\lambda_{p}$ and its inverse $\lambda_{p}^{-1}$ in $\pi_{1}(S ; p)$, respectively. It was also proved in [17] that

$$
[\gamma, \lambda]=\frac{1}{2} \sum_{p \in \gamma \# \lambda} \varepsilon(p ; \gamma, \lambda)\left(\gamma_{p} \lambda_{p}-\gamma_{p} \lambda_{p}^{-1}\right)
$$

defines a Lie bracket on $\mathbb{R} \hat{\pi}$.

Goldman's Lie bracket was generalized by Chas and Sullivan to a bracket on the equivariant homology $H_{*}^{S^{1}}(L M)$ of the free loop space $L M$ of an oriented closed manifold $M$ of arbitrary finite dimension $m$ :

$$
[\cdot, \cdot]: H_{i}^{S^{1}}(L M) \otimes H_{j}^{S^{1}}(L M) \rightarrow H_{i+j+2-m}^{S^{1}}(L M) .
$$

This makes $H_{*}^{S^{1}}(L M)$ into a graded Lie algebra, after a shift by $m-2$ in the grading. For an oriented surface $M$ of dimension $m=2$ and $i=j=0$, the bracket on $\mathbb{R} \hat{\pi}=H_{0}^{S^{1}}(L M)$ coincides with that discovered by Goldman.

Inspired by Goldman [17] and Cattaneo, Frohlich and Pedrini [3], we do something similar for the Chas-Sullivan bracket in this paper. More precisely, let $G$ denote $\operatorname{GL}(n, \mathbb{R})$ or $\operatorname{GL}(n, \mathbb{C})$, endowed with the invariant function $f(g)=\operatorname{Re} \operatorname{tr}(g)$, and 
$\mathfrak{g}$ its Lie algebra with the nondegenerate invariant bilinear form $\langle x, y\rangle=\operatorname{Re} \operatorname{tr}(x y)$. Let $G \rightarrow P \rightarrow M$ be a principal bundle over a compact and oriented manifold $M$ of dimension $m=2 d$, with a fixed flat connection $\nabla$. We construct a map of Lie algebras $\Psi$ from the equivariant homology $H_{2 *}^{S^{1}}(L M)$ to the Poisson algebra of function on the symplectic space $\mathcal{M C}$, which is the formal completion of the space $\left\{x \in \bigoplus_{k \geq 0} \Omega^{2 k+1}(M, \operatorname{ad} P) \mid d_{\nabla} x+1 / 2[x, x]=0\right\} / \mathcal{G}$ (see Section 4 for definition) of the differential graded Lie algebra $\left(\Omega^{*}(M, \operatorname{ad} P), d_{\nabla}\right)$ of differential forms with values in $\operatorname{ad} P$, the associated adjoint bundle of $P$ (see Gan and Ginzburg [13]). For a description of the natural symplectic structure of $\mathcal{M C}$ see Example 4.2, Proposition 4.3 and Theorem 4.4. Note that using $\nabla$ as a point of reference, the Maurer-Cartan moduli space contains a copy of the moduli space of flat connection on $G \rightarrow P \rightarrow M$ (see the discussion in Section 9). Here is one of the main theorems:

Theorem 7.4 For $G=\operatorname{GL}(n, \mathbb{C})$ or $\operatorname{GL}(n, \mathbb{R})$, the generalized holonomy map,

$$
\Psi:\left(H_{2 *}^{S^{1}}(L M),[\cdot, \cdot]\right) \rightarrow(\mathcal{O}(\mathcal{M C}),\{\cdot, \cdot\}),
$$

is a map of Lie algebras.

For a definition of generalized holonomy see Section 6 (and Cattaneo and Rossi [4]). For a loop $\lambda \in L M$, representing an element of $H_{0}^{S^{1}}(L M)$, the value of the function $\Psi_{\lambda}$ at a flat connection is the trace of its holonomy along $\lambda$. The machinery of the proof is robust enough to handle other reductive subgroups of $\operatorname{GL}(n, \mathbb{C})$. In a manner similar to the discussion in [17], different subgroups correspond different Lie algebra structures on the equivariant homology of free loop spaces. We discuss the cases of $G=\mathrm{O}(p, q), \mathrm{O}(n, \mathbb{C}), \mathrm{U}(p, q), \mathrm{Sp}(n, \mathbb{R})$, and $\mathrm{Sp}(p, q)$ (see Section 8).

We provide a mathematician-friendly formulation and proof of the main result of [3] for $G=\operatorname{GL}(n, \mathbb{C})$ and $\operatorname{GL}(n, \mathbb{R})$ together with its natural generalization to other reductive Lie groups. The relevance of the subject matter to the Chern-Simons theory and its applications are explained by Schwarz [24].

Let us very briefly review each section. Section 2 is a short description of the loop product and string bracket. In Section 3 we recall basic facts about invariant functions on Lie groups and some of their byproducts. Section 4 discusses the symplectic nature of the set of all solutions of the Maurer-Cartan equation on a differential graded algebra. We discuss the symplectic structure of the moduli space of Maurer-Cartan equation via the process of Hamiltonian reduction. Section 6 contains the main part of the paper. The main concept here is that of the generalized holonomy. Section 7 concerns the construction of the Lie algebra map for $G=\operatorname{GL}(n, \mathbb{C})$ or $\operatorname{GL}(n, \mathbb{R})$. Section 8 deals with several other linear reductive Lie groups which tie with the Lie algebra 
structure on the vector space based on free homotopy classes of unoriented curves. Section 9 explains how for a 2-dimensional manifold our construction specializes to that described by Goldman [17]. In Appendix A, we recall the moduli space of flat connections and its relation to the moduli space of representations of the fundamental group. We also review the basic facts about homology and cohomology with local coefficients as well as a relevant version of Poincaré duality. Appendix B describes the formula for the solutions of the time dependent linear system of equations in terms of the Chen iterated integrals.

Acknowledgments The authors would like to thank Jean Barge, David Chataur, Alberto Cattaneo, Jean Lannes, Riccardo Longoni, and Dennis Sullivan for many helpful conversations and comments. We are grateful to Carlo A Rossi and James Stasheff who read the first version of the paper and suggested many important corrections and improvements. We are also thankful to Victor Ginzburg and John Terrila for their useful suggestions regarding the algebraic treatment of the moduli space. Referees' comments have helped put this work on more solid technical grounds; for them we are thankful.

The first author was supported by a ChateauBriand Postdoctoral Fellowship while he was visiting École Polytechnique 2004-2005.

\section{String bracket}

Let $M$ be an closed oriented manifold of dimension $m$. In [5; 6], Chas and Sullivan forged the term String Topology by introducing various operations on the ordinary and equivariant homologies of $L M=C^{\infty}\left(S^{1}, M\right)$ the free loop space of $M$, where $S^{1}=\mathbb{R} / \mathbb{Z}$. The free loop space $L M$ is therefore a Fréchet manifold benefiting from such tools as the differential forms, principal bundles, connection, and others (see Section 6 for more details). Throughout this paper the coefficient ring of the (co)homology is $\mathbb{Z}$ unless otherwise it is specified.

Chas and Sullivan's constructions includes a product on $H_{*+m}(L M)$, called the loop product, and its equivariant version, the string bracket, defined on the shifted $S^{1}$ equivariant homology $H_{*+m-2}^{S^{1}}(L M)=H_{*+m-2}\left(E S^{1} \times_{S^{1}} L M\right)$. For the purposes of this paper we use a description of the loop product found in Cohen and Jones [10]. Let $L M \times_{M} L M=\left\{\left(\gamma_{1}, \gamma_{2}\right) \mid \gamma_{1}(0)=\gamma_{2}(0)\right\} \subset L M \times L M$ be the space of the pairs of loops with the identical marked points and consider the following commutative

\footnotetext{
${ }^{1}$ In the literature $L M$ is $C^{0}\left(S^{1}, M\right)$ which has the same homotopy type of $C^{\infty}\left(S^{1}, M\right)$.
} 
diagram:

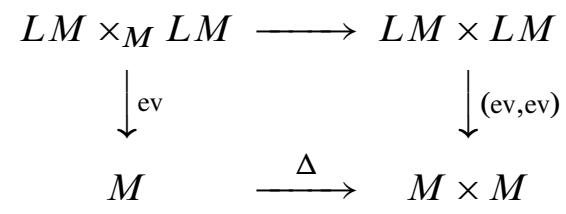

Note that $L M \times \times_{M} L M$ is a codimension $m$ subspace of $L M \times L M$ with a tubular neighborhood $\mathrm{ev}^{*}\left(v_{M}\right)$ where $v_{M}$ is a normal bundle for the diagonal $M \hookrightarrow M \times M$.

Consider the map

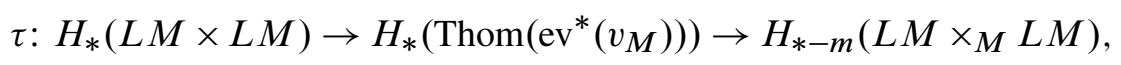

where the first map is Pontrjagin-Thom collapsing map to the Thom space of ev* $\left(v_{M}\right)$, and the second is the Thom isomorphism for the normal bundle $\operatorname{ev}^{*}\left(v_{M}\right)$. Also, consider the map

$$
\varepsilon: H_{*}\left(L M \times_{M} L M\right) \rightarrow H_{*}(L M)
$$

induced by the concatenation of the loops $\lambda_{1}$ and $\lambda_{2}$ with the identical marked points:

$$
\lambda_{1} \circ \lambda_{2}(t)= \begin{cases}\lambda_{1}(2 t) & 0 \leq t \leq 1 / 2 \\ \lambda_{2}(2 t-1) & 1 / 2 \leq t \leq 1\end{cases}
$$

The loop product is defined as follows:

$$
\bullet=\varepsilon \circ \tau: H_{*}(L M) \otimes H_{*}(L M) \simeq H_{*}(L M \times L M) \rightarrow H_{*-m}(L M) .
$$

Remark 2.1 Here we have to modify the definition of $\lambda_{1} \circ \lambda_{2}$ since the result may not be smooth. For that one has to reparameterized the $\lambda_{1}$ and $\lambda_{2}$ in a neighborhood of 0 using a fixed smooth bijection of $[0,1]$ whose all derivatives at 0 and 1 are zero. This is standard and does not change the homological operations introduced above.

Next, we recall the definition of the string bracket on $H_{*+m-2}^{S^{1}}(L M)$ [5]. Consider the degree one $S^{1}$-transfer map $\mathfrak{m}_{*}: H_{*}^{S^{1}}(L M) \rightarrow H_{*+1}(L M)$, and the degree zero map $\mathfrak{e}_{*}: H_{*}(L M) \rightarrow H_{*}^{S^{1}}(L M)$ induced by the projection $L M \times E S^{1} \rightarrow\left(L M \times{ }_{S^{1}} E S^{1}\right)$ $[12 ; 11]$. The string bracket $[\cdot, \cdot]: H_{i}^{S^{1}}(L M) \otimes H_{j}^{S^{1}}(L M) \rightarrow H_{i+j+2-m}^{S^{1}}(L M)$ is defined as follows:

$$
[a, b]=(-1)^{|a|} \mathfrak{e}_{*}\left(\mathfrak{m}_{*} a \bullet \mathfrak{m}_{*} b\right) .
$$

It was proved in Chas and Sullivan [5] that $\left(H_{*+m-2}^{S^{1}}(L M),[\cdot, \cdot]\right)$ is a graded Lie algebra and that $\Delta=\mathfrak{m}_{*} \circ \mathfrak{e}_{*}: H_{*+m}(L M) \rightarrow H_{*+m+1}(L M)$ together with the loop product makes $H_{*+m}(L M)$ into a $B V$ algebra. In fact $\Delta$ is the map induced by the unit circle action. 
Throughout this paper $M$ is a manifold of dimension $m=2 d$ and therefore the shift in the degrees by the dimension does not change the parity. This means $H_{2 *}^{S^{1}}(L M)$ may simply be regarded as a nongraded Lie algebra. In this case the equation (2-1) becomes

$$
[a, b]=\mathfrak{e}_{*}\left(\mathfrak{m}_{*} a \bullet \mathfrak{m}_{*} b\right)
$$

for $a, b \in H_{2 *}^{S^{1}}(L M)$.

\section{Invariant functions and principal bundles}

In this section, we briefly recall some basic facts and definitions. Most of the material is taken from Goldman [17]. Let $G$ be a Lie group with Lie algebra $\mathfrak{g}$. Assume that $\mathfrak{g}$ is equipped with a nondegenerate bilinear form $\langle\cdot, \cdot\rangle$, which is invariant, that is, $\langle[x, y], z\rangle=\langle x,[y, z]\rangle$, for all $x, y, z \in \mathfrak{g}$. We think of an element $x \in \mathfrak{g}$ as a left invariant derivation on $C^{\infty}(G)$ defined by

$$
(x \cdot f)(g)=\left.\frac{d}{d t} f(g \exp (t x))\right|_{t=0},
$$

for $x \in \mathfrak{g}$. The universal enveloping algebra $U \mathfrak{g}=\bigoplus_{k=0}^{\infty} \mathfrak{g}^{\otimes k} /\langle a \otimes b-b \otimes a-[a, b]\rangle$ may then be regarded as the associative algebra of all left invariant differential operators on $C^{\infty}(G)$. An invariant function $f: G \rightarrow \mathbb{R}$ is a $C^{\infty}$ function which is invariant under conjugation. The variation function of an invariant function $f: G \rightarrow \mathbb{R}$, with respect to a nondegenerate invariant bilinear form $\langle\cdot, \cdot\rangle$, is the map $F: G \rightarrow \mathfrak{g}$ defined by

$$
\langle F(g), x\rangle=(x \cdot f)(g) .
$$

Note that $F$ is a $G$-equivariant map with respect to the conjugation action on the domain and the adjoint action on the range. In fact, one may extend $F: G \rightarrow \mathfrak{g}$ to $\widehat{F}: G \times U \mathfrak{g} \rightarrow \mathfrak{g}$ (using the identification $G \cong G \times\{1\} \subset G \times U \mathfrak{g}$ ) as follows.

$$
\widehat{F}\left(g ;\left[x_{1} \otimes \cdots \otimes x_{k}\right]\right)=\left.\frac{\partial^{k}}{\partial t_{1} \cdots \partial t_{k}} F\left(g \exp \left(t_{1} x_{1}\right) \cdots \exp \left(t_{k} x_{k}\right)\right)\right|_{(0, \cdots, 0)} .
$$

We will later, in equations (3-5) and (3-6), apply the above to fibers of appropriate bundles.

A case of particular interest is when $G \subset \mathrm{GL}(n, \mathbb{C})$ is a reductive subgroup, that is, a closed subgroup which is invariant under the operation of conjugate transpose. It is evident that $f(g)=\operatorname{Re} \operatorname{tr} \rho(g)$ is an invariant function. Moreover, reductivity implies that the invariant bilinear form $\langle x, y\rangle=\operatorname{Re} \operatorname{tr}(\rho(x) \rho(y))$ is in fact nondegenerate. In fact, slightly more generally, any covering of such a $G$ would enjoy the above invariant function and bilinear form. 
Proposition 3.1 Let $G \subset \mathrm{GL}(n, \mathbb{C})$ be a reductive subgroup endowed with the invariant function $f(g)=\operatorname{Re} \operatorname{tr} \rho(g)$. The variation function of $f$ with respect to the nondegenerate invariant form $\langle x, y\rangle=\operatorname{Re} \operatorname{tr}(\rho(x) \rho(y))$ is the composition

$$
G \stackrel{\rho}{\rightarrow} \mathrm{GL}(n, \mathbb{C}) \stackrel{i}{\hookrightarrow} \mathfrak{g l}(n, \mathbb{C}) \stackrel{\mathrm{pr}}{\rightarrow} \mathfrak{g},
$$

where $i$ is the inclusion of $n \times n$ invertible matrices in all $n \times n$ matrices and $\mathrm{pr}$ is the orthogonal projection onto $\mathfrak{g}$ with respect to $\langle\cdot, \cdot\rangle$.

Corollary 3.2 For $G=\operatorname{GL}(n, \mathbb{R})$ or $\operatorname{GL}(n, \mathbb{C})$, endowed with the invariant function $f(g)=\operatorname{Re} \operatorname{tr}(g)$ and invariant bilinear form $\langle x, y\rangle=\operatorname{Re} \operatorname{tr}(x y)$, the variation function $F: \operatorname{GL}(n, \mathbb{F}) \rightarrow \mathfrak{g l}(n, \mathbb{F})$ is the inclusion map of invertible matrices in all real and complex matrices, respectively. Moreover, for $k \geq 1$,

$$
\widehat{F}\left(g ;\left[x_{1} \otimes \cdots \otimes x_{k}\right]\right)=g x_{1} \cdots x_{k} .
$$

Corollary 3.3 Let $G=\mathrm{O}(p, q), \mathrm{O}(n, \mathbb{C}), \mathrm{U}(p, q), \operatorname{Sp}(n, \mathbb{R})$ and $\operatorname{Sp}(p, q)$, endowed with the invariant function $f(g)=\operatorname{Re} \operatorname{tr}(g)$ and invariant bilinear form $\langle x, y\rangle=$ $\operatorname{Re} \operatorname{tr}(x y)$. Then, the variation function $F: G \rightarrow \mathfrak{g}$ is given by $F(g)=\frac{1}{2}\left(g-g^{-1}\right)$. Moreover, for $k \geq 1$,

$$
\widehat{F}\left(g ;\left[x_{1} \otimes \cdots \otimes x_{k}\right]\right)=\frac{1}{2} g x_{1} \cdots x_{k}+\frac{(-1)^{k+1}}{2} x_{k} \cdots x_{1} g^{-1} .
$$

The action of the universal enveloping algebra $U \mathfrak{g}$ on $C^{\infty}(G)$ gives rise to an extension $\widehat{f}: G \times U \mathfrak{g} \rightarrow \mathbb{R}$ of $f: G \rightarrow \mathbb{R}$ (using the identification $G \cong G \times\{1\} \subset G \times U \mathfrak{g}$ ) defined by

$$
\begin{aligned}
\hat{f}(g, r) & =r f(g), \quad \text { for } r \in \mathbb{R} \\
\hat{f}\left(g ;\left[x_{1} \otimes \cdots \otimes x_{k+1}\right]\right) & =\left(\left[x_{1} \otimes \cdots \otimes x_{k+1}\right] \cdot f\right)(g) \\
& =\left.\frac{\partial^{k+1}}{\partial t_{1} \cdots \partial t_{k+1}} f\left(g \exp \left(t_{1} x_{1}\right) \cdots \exp \left(t_{k+1} x_{k+1}\right)\right)\right|_{(0, \cdots, 0)} .
\end{aligned}
$$

We have

$$
\begin{aligned}
& \hat{f}\left(g ;\left[x_{1} \otimes \cdots \otimes x_{k+1}\right]\right) \\
& =\left.\frac{\partial^{k}}{\partial t_{1} \cdots \partial t_{k}}\left\langle\hat{F}\left(g \exp \left(t_{1} x_{1}\right) \cdots \exp \left(t_{k} x_{k}\right)\right), x_{k+1}\right\rangle\right|_{(0, \cdots, 0)} .
\end{aligned}
$$

For the invariant function $\operatorname{tr}: \operatorname{GL}(n, \mathbb{C}) \rightarrow \mathbb{R}, f(g)=\operatorname{Re} \operatorname{tr}(g)$, and the bilinear form $\langle x, y\rangle=\operatorname{Re} \operatorname{tr}(x y)$, we have

$$
\widehat{\operatorname{tr}}\left(g ;\left[x_{1} \otimes \cdots \otimes x_{k+1}\right]\right)=\operatorname{tr}\left(g x_{1} \cdots x_{k+1}\right) .
$$


Let $M$ be a closed oriented manifold of dimension $m=2 d$ and $G \rightarrow P \rightarrow M$ be a principal bundle equipped with a flat connection $\nabla$. Let $P(b)$ denote the fiber of $P$ at a point $b \in M$. Let conj: $G \rightarrow \operatorname{Aut}(G)$ denote the action of $G$ on itself defined by $\operatorname{conj}(g)(h)=g^{-1} h g$. Since conjugation fixes the identity, conj induces an action Ad: $G \rightarrow \operatorname{Aut}(\mathfrak{g})$ and subsequently an action $\operatorname{Ad}: G \rightarrow \operatorname{Aut}(U \mathfrak{g})$. Let $P_{G}$, ad $P$, and $P_{\mathfrak{u}}$ respectively denote the associated bundles to these representation, with fibers $P_{G}(b), \operatorname{ad} P(b)$, and $P_{\mathfrak{u}}(b)$ at a point $b$. Then, $P_{G}(b)$ is canonically identified as the group of $G$-equivariant diffeomorphisms of the fiber $P(b)$ of $P$ at that point $b$. This group is isomorphic to $G$, while there is no natural choice of an isomorphism. The set of all sections of $P_{G}$, denoted by $\Gamma\left(P_{G}\right)$, is then identifiable as the group of fiber preserving and $G$-equivariant diffeomorphisms of $P$. By the same token the adjoint representation Ad: $G \rightarrow \operatorname{Aut}(\mathfrak{g})$ gives rise to an associated vector bundle ad $P$ whose fiber ad $P(b)$ is a Lie algebra canonically identified with the Lie algebra of $P_{G}(b)$. Clearly, there is a well-defined exponential map exp: ad $P \rightarrow P_{G}$ inducing and exponential map exp: $\Gamma(\operatorname{ad} P) \rightarrow \Gamma\left(P_{G}\right)$ and there are natural actions conj: $P_{G}(b) \times \operatorname{ad} P(b) \rightarrow \operatorname{ad} P(b)$ and conj: $P_{G}(b) \times P_{\mathfrak{u}}(b) \rightarrow P_{\mathfrak{u}}(b)$ inducing an actions Ad: $\Gamma\left(P_{G}\right) \times \Gamma(\operatorname{ad} P) \rightarrow \Gamma(\operatorname{ad} P)$ and Ad: $\Gamma\left(P_{G}\right) \times \Gamma\left(P_{\mathfrak{u}}\right) \rightarrow \Gamma\left(P_{\mathfrak{u}}\right)$. Therefore there is a natural associative multiplication, $*$, on $P_{G}(b) \times P_{\mathfrak{u}}(b)$ for every $b$ defined as follows:

$$
(g, u) *(h, v)=\left(g h, \operatorname{Ad}_{h}(u) \otimes v\right) .
$$

Here Ad is the induced action of the group $G$ on the universal enveloping algebra, $U \mathfrak{g}$, of its Lie algebra $\mathfrak{g}$.

This multiplication extends naturally to $\Gamma\left(P_{G}\right) \times \Omega^{*}\left(M, P_{\mathfrak{u}}\right)$. For $i \in\{1,2\}$, if $\left(h_{i}, \alpha_{i}\right) \in \Gamma\left(P_{G}\right) \times \Omega^{*}\left(M, P_{\mathfrak{u}}\right)$,

$$
\left(h_{1}, \alpha_{1}\right) *\left(h_{2}, \alpha_{2}\right)=\left(h_{1} h_{2},\left(\operatorname{Ad}_{h_{2}} \alpha_{1}\right) \wedge \alpha_{2}\right),
$$

where $\wedge$ product on $\Omega\left(M, P_{\mathfrak{u}}\right)$ is the tensor product of two multiplications: the exterior product of differential forms and the multiplication of values in $P_{\mathfrak{u}}$. Note that unlike the ordinary wedge product which is associative and graded commutative, $\wedge$ is only associative.

An invariant function $f: G \rightarrow \mathbb{R}$ induces a function $f: P_{G} \rightarrow \mathbb{R}$, and subsequently $f: \Gamma\left(P_{G}\right) \rightarrow C^{\infty}(M)$. Also, note that $\hat{f}$ extends to

$$
\widehat{f}: \Gamma\left(P_{G}\right) \otimes \Gamma\left(P_{\mathfrak{u}}\right) \rightarrow C^{\infty}(M) .
$$

Similarly, $\hat{F}: G \times U \mathfrak{g} \rightarrow \mathfrak{g}$ naturally extends to

$$
\widehat{F}: \Gamma\left(P_{G}\right) \otimes \Gamma\left(P_{\mathfrak{u}}\right) \rightarrow \Gamma(\operatorname{ad} P) .
$$


Remark 3.4 For linear groups $G=\mathrm{GL}(n, \mathbb{C})$ or $\operatorname{GL}(n, \mathbb{R})$, the Lie algebra $\mathfrak{g}$ is actually an associative algebra, and therefore there is a natural map of associative algebras $\Pi: U \mathfrak{g} \rightarrow \mathfrak{g}$, sending $\Pi:\left[x_{1} \otimes \cdots \otimes x_{k}\right] \mapsto x_{1} \cdots x_{k}$. Moreover, for tr: $G \rightarrow \mathbb{R}$ and $\langle x, y\rangle=\operatorname{Re} \operatorname{tr}(x y)$, one can show that

$$
\begin{aligned}
\hat{\operatorname{tr}}\left(( h _ { 1 } , [ x _ { 1 } \otimes \cdots \otimes x _ { k } ] ) * \left(h_{2},\left[y_{1} \otimes \cdots\right.\right.\right. & \left.\left.\left.\otimes y_{l}\right]\right)\right) \\
& =\operatorname{tr}\left(h_{1} \Pi\left[x_{1} \otimes \cdots \otimes x_{k}\right] h_{2} \Pi\left[y_{1} \otimes \cdots \otimes y_{l}\right]\right) \\
& =\operatorname{tr}\left(h_{1} x_{1} \cdots x_{k} h_{2} y_{1} \cdots y_{l}\right),
\end{aligned}
$$

where in the last line tr: $\mathfrak{g} \rightarrow \mathbb{R}$ is really the derivative of tr: $G \rightarrow \mathbb{R}$ at the identity. This gives rise to the following identity for elements $\alpha_{1}, \alpha_{2} \in \Omega^{*}\left(M, P_{\mathfrak{u}}\right)$ :

$$
\widehat{\operatorname{tr}}\left(\left(h_{1}, \alpha_{1}\right) *\left(h_{2}, \alpha\right)\right)=\operatorname{tr}\left(h_{1} \Pi \alpha_{1} \wedge h_{2} \Pi \alpha_{2}\right),
$$

where tr: $\Omega^{*}(M, \operatorname{ad} P) \rightarrow C^{\infty}(M)$ and in the right hand side all multiplication are matrix multiplications.

\section{Symplectic nature of the Maurer-Cartan moduli space}

In this section, we mostly follow Gan and Ginzburg [13]. For the sake of completeness and clarity we mention some of their result without repeating their proofs.

Definition 4.1 A cyclic differential graded Lie algebra, $(\mathcal{L}, d,[\cdot, \cdot], \omega(\cdot, \cdot))$, or a cyclic DGLA for short, consists of a $\mathbb{Z}_{2}$-graded Lie algebra $\mathcal{L}=\mathcal{L}_{0} \oplus \mathcal{L}_{1}$, with a differential $d$, and a bilinear form $\omega: \mathcal{L} \times \mathcal{L} \rightarrow \mathbb{R}$, satisfying:

(i) $d\left(\mathcal{L}_{0}\right) \subseteq \mathcal{L}_{1}$ and $d\left(\mathcal{L}_{1}\right) \subseteq \mathcal{L}_{0}$

(ii) $d[x, y]=[d x, y]+(-1)^{|x|}[x, d y]$

(iii) $\omega([x, y], z)=\omega(x,[y, z])$

(iv) $\omega(d x, y)+(-1)^{|x|} \omega(x, d y)=0$

(v) $\omega(y, x)=(-1)^{|x||y|} \omega(x, y)$

(vi) $\left.\omega(\cdot, \cdot)\right|_{\mathcal{L}_{0}}$ and $\left.\omega(\cdot, \cdot)\right|_{\mathcal{L}_{1}}$ are nondegenerate

(vii) $\omega(x, y)=0$ for $x \in \mathcal{L}_{0}$ and $y \in \mathcal{L}_{1}$

We furthermore assume that $\mathcal{L}_{0}$ is the Lie algebra of a complex, connected, and simply connected linear algebraic group $\mathcal{G}$. 
Example 4.2 Let $G \rightarrow P \rightarrow M$ be a principal bundle over a compact and oriented manifold $M$ without boundary of dimension $m=2 d$ which is endowed with a flat connection $\nabla$. Assume that the Lie algebra, $\mathfrak{g}$, of $G$ is equipped with a nondegenerate invariant bilinear form $\langle\cdot, \cdot\rangle$. Recall that invariance means $\langle[x, y], z\rangle=\langle x,[y, z]\rangle$. Consider the graded Lie algebra $\mathcal{L}=\Omega^{*}(M, \operatorname{ad} P)$ of differential forms with values in $\operatorname{ad} P$, the adjoint bundle of $P$. In order to see the graded Lie algebra structure better, recall that the tensor product of a graded commutative algebra and a graded Lie algebra is a graded Lie algebra. Note that $\mathcal{L}=\Omega^{*}(M, \operatorname{ad} P)=\Omega^{*}(M) \otimes_{C}{ }_{(M)} \Gamma(\operatorname{ad} P)$. Here, the graded Lie algebra $\Gamma(\operatorname{ad} P)$ is concentrated in degree zero. The covariant derivative associated to the connection $\nabla$ induces a derivation $d_{\nabla}: \Omega^{*}(M, \operatorname{ad} P) \rightarrow$ $\Omega^{*+1}(M, \operatorname{ad} P)$. Flatness means that $d_{\nabla}$ is a differential, that is to say $d_{\nabla}^{2}=0$. Define a bilinear form $\omega: \mathcal{L} \times \mathcal{L} \rightarrow \mathbb{R}$ as the following composition:

$\omega: \Omega^{i}(M, \operatorname{ad} P) \times \Omega^{j}(M, \operatorname{ad} P) \stackrel{\wedge}{\rightarrow} \Omega^{i+j}(M, \operatorname{ad} P \otimes \operatorname{ad} P) \stackrel{\langle\cdot, \cdot\rangle}{\longrightarrow} \Omega^{i+j}(M, \mathbb{R}) \stackrel{\int_{M}}{\longrightarrow} \mathbb{R}$, if $i+j=2 d$, and zero otherwise.

Clearly, conditions (i) and (iii) are satisfied and conditions (ii) and (iv) follow from Corollary A.2 and Corollary A.3. It follows from the invariance property and nondegeneracy of $\langle\cdot, \cdot\rangle$ that conditions (v)-(vi) hold. Lastly, (vii) is true because the manifold is even dimensional. This means $\Omega^{*}(M, \operatorname{ad} P)$ is a cyclic DGLA.

Given a cyclic DGLA $(\mathcal{L}, d,[\cdot, \cdot], \omega(\cdot, \cdot))$, clearly $\left(\mathcal{L}_{1},\left.\omega\right|_{\mathcal{L}_{1}}\right)$ is a symplectic vector space. To every $a \in \mathcal{L}_{0}$, one associates a vector field on $\mathcal{L}_{1}$ by defining $\xi_{a}(x)=$ $[a, x]-d a$, for all $x \in \mathcal{L}_{1}$. The vector field $\xi_{a}$ respects the symplectic structure, that is to say, $L_{\xi_{a}} \omega=0$. In fact, this infinitesimal action is Hamiltonian and the following proposition describes its moment map. For a discussion and proof see Section 1.5 of [13].

Proposition 4.3 Let $(\mathcal{L}, d,[\cdot, \cdot], \omega)$ be a cyclic DGLA. The map $\phi: \mathcal{L}_{1} \rightarrow \mathcal{L}_{0}^{*}$ defined by $\phi(x)(a)=\omega(d x+1 / 2[x, x], a)$, for all $x \in \mathcal{L}_{1}$ and $a \in \mathcal{L}_{0}$, is a moment map for the action of $\mathcal{L}_{0}$ on the symplectic space $\left(\mathcal{L}_{1},\left.\omega\right|_{\mathcal{L}_{1}}\right)$.

Define $\quad \operatorname{MC}(\mathcal{L})=\left\{x \in \mathcal{L}_{1} \mid d x+1 / 2[x, x]=0\right\}$.

Note that the map $a \mapsto \xi_{a}$ is a Lie algebra homomorphism and that for every $a \in \mathcal{L}_{0}$, the vector field $\xi_{a}$ is tangent to $\operatorname{MC}(\mathcal{L})$ [13, Lemma 1.2.1]. This implies a well-defined action of $\mathcal{G}$ on $\mathcal{L}_{1}$ by affine linear transformations referred to as the gauge group. Note that this action is different than the ordinary adjoint action of $\mathcal{G}$ on $\mathcal{L}_{1}$ and it keeps invariant the scheme $\operatorname{MC}(\mathcal{L})$. 
Let us be more precise about the nature of the quotient $\operatorname{MC}(\mathcal{L}) / \mathcal{G}$. The space $\operatorname{MC}(\mathcal{L})$ is a defined as the zero level set of the moment map. Since the group $\mathcal{G}$ keeps $\operatorname{MC}(\mathcal{L})$ invariant, one can consider the quotient $\mathrm{MC}(\mathcal{L}) / \mathcal{G}$. Note that because 0 is not a regular value and $\mathcal{G}$ does not act freely, $\operatorname{MC}(\mathcal{L}) / \mathcal{G}$ is not a smooth variety. The good news is that just as in [13] one may still treat $\operatorname{MC}(\mathcal{L}) / \mathcal{G}$ completely algebraically as a formal scheme [13, Remark 2.2.1]. Thus, one starts with a formal completion $\hat{\mathcal{L}}_{1}$ of $\mathcal{L}_{1}$ at the origin and considers the closed subscheme $\phi^{-1}(0) \subset \widehat{\mathcal{L}}_{1}$. Then, the completion of $\mathcal{G}$ gives a pro-algebraic groupoid which acts on $\phi^{-1}(0)$. Passing to the quotient yields a pro-algebraic stack $\mathcal{M C}(\mathcal{L})$. We use $\mathcal{O}(\mathcal{M C}(\mathcal{L}))$ to denote the coordinate ring of $\mathcal{M C}(\mathcal{L})$. Thus, elements of $\mathcal{O}(\mathcal{M C}(\mathcal{L}))$ have representatives in $\mathcal{O}\left(\widehat{\mathcal{L}}_{1}\right)=\mathbb{C}\left[\left[\widehat{\mathcal{L}}_{1}\right]\right]$, the formal power series on $\mathcal{L}_{1}$. In the best of all cases one may be able to show that certain desired series converge. This is in fact what happens in our definition of the generalized holonomy (see (6-2) and Definition 6.1) as explained in Remark B.2. Let us also talk about the symplectic nature of the moduli space. In the smooth case, where one deals with the pre-image of a regular value of the moment map and has a free group action, the symplectic quotient is a smooth variety with a well-defined Zarisky tangent space at each point. Unfortunately, this is hardly the case in almost every interesting example. In such cases, one can talk about the tangent space $T_{p} \mathcal{M C}$ as a 3 -term complex $\operatorname{Lie}(\mathcal{G}) \rightarrow T_{p} \widehat{\mathcal{L}}_{1} \rightarrow \operatorname{Lie}(\mathcal{G})^{*}$, concentrated in degrees $-1,0$, and 1 , where $\operatorname{Lie}(\mathcal{G})$ is the Lie algebra of $\mathcal{G}$ [13, Section 1.6]. It is clear that only in the nicest of all cases, when 0 is a regular value of the moment map and $\mathcal{G}$ acts freely, that the homology of this complex is concentrated in degree 0 . In general, one needs to think about this complex as the tangent space (complex) to the symplectic reduction $\mathcal{M C}(\mathcal{L})$ at a point $p$, keeping track of all the failures. In this enlightened view, a symplectic structure on $\mathcal{M C}(\mathcal{L})$, for instance, is a isomorphism $\omega_{p}: T_{p} \mathcal{L}_{1} \rightarrow T_{p}^{*} \mathcal{L}_{1}$, establishing an isomorphism between the tangent complex and its dual. The symplectic structure on $\mathcal{M C}$ gives $\mathcal{O}(\mathcal{M C}(\mathcal{L}))$ a Poisson algebra structure.

The following is a direct application of a theorem of Gan and Ginzburg [13] for a reductive subgroup $G \subseteq \mathrm{GL}(n, \mathbb{C})$ equipped with the nondegenerate invariant bilinear form $\langle x, y\rangle=\operatorname{Re} \operatorname{tr}(x y)$.

Theorem 4.4 Let $G \subseteq \mathrm{GL}(n, \mathbb{C})$ be a reductive subgroup and $G \rightarrow P \rightarrow M$ be a principal bundle endowed with a flat connection $\nabla$. The 2-form $\omega(x, y)=\int_{M} \operatorname{Re} \operatorname{tr}(x \wedge y)$ defines a symplectic structure on the Maurer-Cartan moduli space, $\mathcal{M C}\left(\Omega^{*}(M, \operatorname{ad} P)\right)$.

For brevity, we denote $\mathcal{M C}$ for the Maurer-Cartan moduli space $\mathcal{M C}\left(\Omega^{*}(M, \operatorname{ad} P)\right)$. Note that a typical element in $\mathcal{M C}$ is a sum of differential forms of odd degrees. One may think of $\mathcal{M C}$ as an extension of the moduli space of flat connections on $P$. In fact, if the Hard Lefschetz theorem holds for $M$, then the moduli space of flat connections 
on $P$ may be viewed as a symplectic substack of $\mathcal{M C}$ (see Section 1.7 of [13] and Section 9 of this paper).

\section{Differential forms on free loop spaces}

In this section we shall present a model of the free loop space $L M$ which is conducive to the notions of the de Rham differential forms and its $S^{1}$-equivariant model. These differential forms contain the Chen iterated integrals [7;8]. Moreover, the cohomology of these forms, called the de Rham cohomology and the equivariant de Rham cohomology respectively, compute the usual and $S^{1}$-equivariant singular cohomologies of $L M$. Such a model of the free loop space must support the usual differential geometric notions such as connections and all related pullback diagrams. Since the different parts of the required framework are developed in different references, we briefly summarize some of the needed definitions and statements and refer the reader to Hamilton [20], Godement [15], Brylinski [2] and Guillemin and Sternberg [19] for a fuller discussion.

Let $E, F$ denote locally convex Hausdorff topological vector space and $U \subset E$ an open set, we say that the map $f: U \rightarrow F$ is of class $C^{1}$ if the limit

$$
d f(x, v)=\lim _{t \rightarrow 0} \frac{f(x+t v)-f(x)}{t}
$$

exists and is continuous as a function of $(x, v) \in U \times E$. Similarly, we can define functions of class $C^{k}$ and thus $C^{\infty}$. Then $\Omega^{n}(U)$ the space of differential $n$-forms on $U$ is defined to be the space of smooth functions $\omega: U \times E^{n} \rightarrow \mathbb{R}$ which are multilinear and antisymmetric in the last $n$ variables. Then, one defines the exterior differential $d$ which satisfies $d^{2}=0$ [20;2]. Also, the Poincaré lemma holds for convex open subsets of $E$ (see Brylinski [2] for a proof).

A differentiable space modeled on $E$ is a Hausdorff space $N$ with a covering $\left\{U_{i}\right\}_{i \in I}$ and a collection of homeomorphisms $\phi_{i}: U_{i} \rightarrow V_{i} \subset E$, such that the transition maps $\phi_{j} \circ \phi_{i}^{-1}$ are smooth. Then a differential $n$-form on an open $U \subset N$ is defined to be a collection of $\omega_{i} \in \Omega^{n}\left(\phi_{i}\left(U \cap U_{i}\right)\right)$ patched together by the transition map $\phi_{j} \circ \phi_{i}^{-1}$. This enables us to define the de Rham complex of $N$ denoted $\left(\Omega^{*}(N), d\right)$ and the de Rham cohomology $H_{D R}^{*}(N)$ of $N$ to be the cohomology of $\left(\Omega^{*}(N), d\right)$.

It is known that the de Rham theorem holds [2]. This is shown using sheaf cohomology. The de Rham forms on the opens $U \subset N$ define a sheaf $\Omega^{*}(N)$ on $N$ which is a resolution of $\underline{\mathbb{R}}_{N}$ the sheaf of the constant functions (by Poincaré lemma). When $N$ is a paracompact differentiable space and the sheaf $\Omega^{*}(N)$ is an acyclic soft sheaf, we can use the natural resolution $\underline{\mathbb{R}}_{N} \rightarrow \underline{\Omega(N)^{*}}$ to calculate the sheaf cohomology $H_{\text {sheaf }}^{*}\left(N, \underline{\mathbb{R}}_{N}\right)$ implying that $H_{D R}^{*}(N) \simeq \overline{H_{\text {sheaf }}^{*}}\left(N, \underline{\mathbb{R}}_{N}\right)[2,1.4 .7 ; 15]$. 
As it turns out, $L M=C^{\infty}\left(S^{1}, M\right)$ can be made into a paracompact differentiable space modeled on $C^{\infty}\left(S^{1}, \mathbb{R}^{n}\right)$ [2, p 110]. The topology of $C^{\infty}\left(S^{1}, \mathbb{R}^{n}\right)$ is defined using a family of the norms $\|\cdot\|_{k}$ given by

$$
\|f\|_{k}^{2}=\int_{0}^{1}\left(\|f(t)\|^{2}+\cdots+\left\|\frac{d^{k}}{d t^{k}} f(t)\right\|^{2}\right) d t,
$$

which make $C^{\infty}\left(S^{1}, \mathbb{R}^{n}\right)$ into a Fréchet vector space and $L M$ into a Fréchet manifold. This allows us to consider vector bundles with connection on $L M$ and do the differential geometry needed in this paper just as in the finite dimensional case (see Hamilton [20] for the details).

Moreover, $C^{\infty}\left(S^{1}, \mathbb{R}^{n}\right)$ can be though of as an inverse limit of the Sobolev spaces $H^{2, k}\left(S^{1}, \mathbb{R}^{n}\right)$, which are the completions of $C^{\infty}\left(S^{1}, \mathbb{R}^{n}\right)$ with respect to the Sobolev norms $\|\cdot\|_{k}$. Therefore, $C^{\infty}\left(S^{1}, \mathbb{R}^{n}\right)$ can be treated as an inverse limit of Hilbert spaces, ILH for short.

Because of the fact that the free loop space $L M$ is modeled on $C^{\infty}\left(S^{1}, \mathbb{R}^{n}\right)$ (which is an ILH) the sheaf $\underline{\Omega(N)^{*}}$ is a soft sheaf, and therefore,

$$
H_{D R}^{*}(L M) \simeq H_{\text {sheaf }}^{*}(L M, \underline{\mathbb{R}} L M) .
$$

It is proved in [15] that for a paracompact topological space $N$,

$$
H_{\text {sheaf }}^{*}\left(N, \mathbb{R}_{N}\right) \simeq H^{*}(N, \mathbb{R}),
$$

therefore,

$$
H_{D R}^{*}(L M) \simeq H^{*}(L M, \mathbb{R}) .
$$

We turn to the equivariant de Rham theorem for which one can find in Guillemin and Sternberg [19] a rigorous treatment for finite dimension manifolds. The same treatment works for $L M$ as we have the right notion of differentials forms for $L M$ with a de Rham theorem. We give the steps whose proofs are exactly as same as the ones in Chapter 2 of [19].

Recall that for a group $G$ acting on a space $N$, the $G$ equivariant cohomology of $\mathrm{N}$ is defined to be $H_{G}^{*}(N, \mathbb{R})=H^{*}(N \times E G / G, \mathbb{R})$ where $E G$ is a contractible topological space on which $G$ acts freely, and $B G=E G / G$ is classifying of the $G$. The case of interest for us is the action of $S^{1}$ on the loop space $L M$ by changing the parametrization. For $S^{k}=\left\{\left.\left(z_{1}, \cdots, z_{k}\right) \in \mathbb{C}^{k}|| z_{1}\right|^{2}+\cdots+\left|z_{k}\right|^{2}=1\right\}$ let

$$
E S^{1}=S^{\infty}=\lim _{\longrightarrow} S^{2 k-1}
$$


defined by the natural inclusions $S^{2 k-1} \rightarrow S^{2 k+1},\left(z_{1}, \cdots, z_{k}\right) \mapsto\left(z_{1}, \cdots, z_{k}, 0\right)$. This comes with the natural inclusions $j_{k}: S^{2 k-1} \rightarrow S^{\infty}$ whose opens are the subset $U \subset S^{\infty}$ with $j_{k}^{-1}(U)$ is open in $S^{2 k-1}$ for all $k$. Then $S^{1}$ acts freely on $S^{\infty}$ and we have $B S^{1}=\mathbb{C} P^{\infty}=\lim \mathbb{C} P^{k-1}$ whose topology is defined similarly. Then the real coefficient equivariant cohomology of $L M$ is defined to be

$$
H_{S^{1}}^{*}(L M, \mathbb{R})=H^{*}\left(L M \times S^{\infty} / S^{1}, \mathbb{R}\right),
$$

where $S^{1}$ acts diagonally on $L M \times S^{\infty}$.

The inclusions $\quad \cdots \rightarrow S^{2 k-1} \rightarrow S^{2 k+1} \rightarrow S^{2 k+3} \rightarrow \cdots$

induce the sequence of the projections

$$
\cdots \leftarrow \Omega^{*}\left(S^{2 k-1}\right) \leftarrow \Omega^{*}\left(S^{2 k+1}\right) \leftarrow \Omega^{*}\left(S^{2 k+3}\right) \leftarrow \cdots,
$$

which allows us to consider to the inverse limit $\Omega^{*}\left(S^{\infty}\right)=\lim \Omega^{*}\left(S^{2 k-1}\right)$ and we can form the complex $\left(\Omega^{*}\left(S^{\infty}\right), d\right)$ which is acyclic and satisfies condition $(\mathrm{C})^{2}$ [19, p 29]. Since $S^{1}$ acts on $L M$ as well as on all $S^{2 k-1}$,s, we can consider the subcomplex of basic forms in $\Omega^{*}(L M) \otimes \Omega^{*}\left(S^{\infty}\right)$, that is the set of all $\omega$ such that

$$
i_{X} \omega=0 \text { and } L_{X} \omega=0,
$$

where $L_{X}=i_{X} \circ d+d \circ i_{X}$ is the Lie derivative. We define the equivariant de Rham cohomology to be

$$
H_{S^{1}, D R}^{*}(L M, \mathbb{R})=H^{*}\left(\left(\Omega^{*}(L M) \otimes \Omega^{*}\left(S^{\infty}\right)\right)_{\mathrm{bas}}\right) .
$$

The rest of this section is devoted to the proof of

$$
H_{S^{1}, D R}^{*}(L M, \mathbb{R}) \simeq H_{S^{1}}^{*}(L M, \mathbb{R}) .
$$

Consider the diagonal action of $S^{1}$ on $L M \times S^{2 k-1}$ and the projection,

$$
\pi: L M \times S^{2 k-1} \rightarrow L M \times S^{2 k-1} / S^{1} .
$$

Since the action is free, the subalgebra $\pi^{*}\left(\Omega^{*}\left(L M \times S^{2 k-1} / S^{1}\right)\right)$ contained in $\Omega^{*}\left(L M \times S^{2 k-1}\right)$ can be characterized as the basic differential forms which we denote $\Omega^{*}\left(L M \times S^{2 k-1}\right)_{\text {bas }}$; moreover $\pi^{*}$ is injective. We denote the subcomplex of basic forms by $\Omega^{*}\left(L M \times S^{2 k-1}\right)$ bas and we have

$$
H_{D R}^{*}\left(L M \times S^{2 k-1} / S^{1}\right) \simeq H^{*}\left(\Omega^{*}\left(L M \times S^{2 k-1}\right) \text { bas }\right) .
$$

\footnotetext{
${ }^{2}$ Condition $(\mathrm{C})$ is an algebraic way of interpreting that an action is locally free.
} 
Using the projections

$$
\cdots \leftarrow \Omega^{*}\left(L M \times S^{2 k-1}\right)_{\text {bas }} \leftarrow \Omega^{*}\left(L M \times S^{2 k+1}\right)_{\text {bas }} \leftarrow \cdots,
$$

we can form the complex $\Omega^{*}\left(L M \times S^{\infty}\right)_{\text {bas }}=\lim _{\longleftarrow} \Omega^{*}\left(L M \times S^{2 k-1}\right)_{\text {bas }}$.

Proposition $5.1 H_{S^{1}}^{*}(L M, \mathbb{R}) \simeq H^{*}\left(\Omega^{*}\left(L M \times S^{\infty}\right)_{\mathrm{bas}}\right)$.

Proof Similarly to the proof of (5-1), for the differentiable space $L M \times S^{2 k-1} / S^{1}$, we have the isomorphism

$$
H_{D R}^{*}\left(L M \times S^{2 k-1} / S^{1}\right)=H^{*}\left(L M \times S^{2 k-1} / S^{1}, \mathbb{R}\right),
$$

which is compatible the inclusions $L M \times S^{2 k-1} / S^{1} \hookrightarrow L M \times S^{2 k+1} / S^{1}$. Therefore,

$$
\begin{aligned}
H_{S^{1}}^{*}(L M, \mathbb{R}) & \simeq \lim _{\longleftarrow} H^{*}\left(L M \times S^{2 k+1} / S^{1}\right) \\
& \simeq \lim _{\longleftarrow} H_{D R}^{*}\left(L M \times S^{2 k-1} / S^{1}\right) \\
& \simeq H^{*}\left(\Omega^{*}\left(L M \times S^{\infty}\right)_{\mathrm{bas}}\right) .
\end{aligned}
$$

So, to prove (5-2) it suffices to show that

$$
H^{*}\left(\Omega^{*}\left(L M \times S^{\infty}\right)_{\mathrm{bas}}\right) \simeq H_{D R}^{*}\left(\left(\Omega^{*}(L M) \otimes \Omega^{*}\left(S^{\infty}\right)\right)_{\mathrm{bas}}\right) .
$$

This follows from a standard spectral sequence argument and the fact that inclusion $\Omega^{*}\left(L M \times S^{\infty}\right) \hookrightarrow \Omega^{*}(L M) \otimes \Omega^{*}\left(S^{\infty}\right)$ induces an isomorphism in cohomology, and that $\Omega^{*}\left(S^{\infty}\right)$ is acyclic and satisfies condition (C) [19, p 30].

\section{Generalized holonomy}

Let $M$ be a compact and oriented manifold without boundary. Given a principal bundle $G \rightarrow P \rightarrow M$ endowed with a flat connection $\nabla$, the trace of the holonomy yields a well-defined map on the set of the free homotopy classes of loops in the base manifold $M$. It was discussed in Cattaneo and Rossi [4] how this may generalize to families of loops, more precisely, to the homology classes in the free loop space of the underlying manifold. We will give a mathematical account of this in this section. Throughout this section we will be using the notion of differential forms developed in Section 5 for $L M$ equipped with it natural Fréchet manifold structure.

For every $n \geq 0$, consider the $n$-simplex,

$$
\Delta^{n}=\left\{\left(t_{0}, t_{1}, \cdots, t_{n}, t_{n+1}\right) \mid 0=t_{0} \leq t_{1} \leq \cdots \leq t_{n} \leq t_{n+1}=1\right\} .
$$


Define the evaluation maps ev and $\mathrm{ev}_{n, i}$, for $1 \leq i \leq n$ as follows:

$$
\begin{aligned}
\text { ev: } \Delta^{n} \times L M & \rightarrow M, & \operatorname{ev}\left(t_{0}, t_{1}, \cdots, t_{n}, t_{n+1} ; \gamma\right) & =\gamma(0)=\gamma(1), \\
\operatorname{ev}_{n, i}: \Delta^{n} \times L M & \rightarrow M, & \operatorname{ev}_{n, i}\left(t_{0}, t_{1}, \cdots, t_{n}, t_{n+1} ; \gamma\right) & =\gamma\left(t_{i}\right) .
\end{aligned}
$$

Let $T_{i}: \mathrm{ev}_{n, i}^{*}(\operatorname{ad} P) \rightarrow \mathrm{ev}^{*}(\operatorname{ad} P)$ denote the map, between pullbacks of the adjoint bundles over $\Delta^{n} \times L M$, defined at a point $\left(0=t_{0}, t_{1}, \cdots, t_{n}, t_{n+1}=1 ; \gamma\right)$ by the parallel transport along and in the direction of $\gamma$ from $\gamma\left(t_{i}\right)$ to $\gamma\left(t_{n+1}\right)=\gamma(1)$, in ad $P$ with respect to the flat connection $\nabla$. Note that if $R, S$, and $T$ denote the parallel transport maps respectively in bundles $P, P_{G}$, and ad $P$, over a given path, then we have $S(\phi)=R^{-1} \circ \phi \circ R$ and $T(x)=d S_{e}(x)$, the derivative at the identity of $S$ evaluated on the vector $x$.

For $\alpha_{i} \in \Omega^{*}(M, \operatorname{ad} P), 1 \leq i \leq n$, define $\alpha(n, i) \in \Omega^{*}\left(\Delta^{n} \times L M, \mathrm{ev}^{*}\right.$ ad $\left.P\right)$ by

$$
\alpha(n, i)=T_{i} \mathrm{ev}_{n, i}^{*} \alpha_{i}
$$

Given $\gamma \in L M$ the holonomy along $\gamma$ from $\gamma(0)$ to $\gamma(1)$ in the principal bundle $P$ gives rise to a section hol $\in \Gamma\left(\mathrm{ev}^{*} P_{G}\right)$. Note that $\Gamma\left(\mathrm{ev}^{*} P_{G}\right)$ acts by conjugation on $\Gamma\left(\mathrm{ev}^{*} P_{G}\right)$, and subsequently on $\Gamma\left(\mathrm{ev}^{*} \mathrm{ad} P\right)$. Define $V_{\alpha_{1}, \cdots, \alpha_{n}}^{n} \in \Omega^{*}\left(L M, \mathrm{ev}^{*} P_{\mathfrak{u}}\right)$ as

$$
\begin{aligned}
& V^{0}=1, \\
& V_{\alpha_{1}, \cdots, \alpha_{n}}^{n}=\int_{\Delta^{n}} \alpha(n, 1) \wedge \cdots \wedge \alpha(n, n), \text { for } n \geq 1,
\end{aligned}
$$

and let

$$
V_{\alpha}=\sum_{n=0}^{\infty} V_{\alpha}^{n}, \text { where } V_{\alpha}^{n}=V_{\alpha, \cdots, \alpha}^{n} .
$$

It is noteworthy that the above infinite sum is convergent. This follows from the discussion in Appendix B, based on the simple fact that the volume of the standard $n$-simplex is $\frac{1}{n !}$ (see Remark B.2).

Then, consider $W_{\alpha_{1}, \cdots, \alpha_{n}}^{n} \in \Gamma\left(\mathrm{ev}^{*} P_{G}\right) \times \Omega^{*}\left(L M, \mathrm{ev}^{*} P_{\mathfrak{u}}\right)$ defined as

$$
W_{\alpha_{1}, \cdots, \alpha_{n}}^{n}=\left(\mathrm{hol}, V_{\alpha_{1}, \cdots, \alpha_{n}}^{n}\right) \text {, for } n \geq 0
$$

and

$$
W_{\alpha}^{n}=\left(\mathrm{hol}, V_{\alpha, \cdots, \alpha}^{n}\right) \text { and } W_{\alpha}=\left(\mathrm{hol}, V_{\alpha}\right) \text {. }
$$

We fix an invariant function $f: G \rightarrow \mathbb{R}$ and we shall follow the notation introduced in (3-6) and (3-1). Note that the maps

$$
\begin{aligned}
& \hat{f}: \Gamma\left(P_{G}\right) \otimes \Gamma\left(P_{\mathfrak{u}}\right) \rightarrow C^{\infty}(M), \\
& \hat{F}: \Gamma\left(P_{G}\right) \otimes \Gamma\left(P_{\mathfrak{u}}\right) \rightarrow \Gamma(\operatorname{ad} P),
\end{aligned}
$$


naturally induce the maps on the differential forms

$$
\begin{aligned}
& \hat{f}: \Gamma\left(P_{G}\right) \otimes \Omega^{*}\left(M, P_{\mathfrak{u}}\right) \rightarrow \Omega^{*}(M), \\
& \hat{F}: \Gamma\left(P_{G}\right) \otimes \Omega^{*}\left(M, P_{\mathfrak{u}}\right) \rightarrow \Omega^{*}(M, \operatorname{ad} P),
\end{aligned}
$$

which in turn induce the following maps on the corresponding pullback bundles:

$$
\begin{aligned}
& \hat{f}: \Gamma\left(\mathrm{ev}^{*} P_{G}\right) \otimes \Omega^{*}\left(L M, \mathrm{ev}^{*} P_{\mathfrak{u}}\right) \rightarrow \Omega^{*}(L M), \\
& \widehat{F}: \Gamma\left(\operatorname{ev}^{*} P_{G}\right) \otimes \Omega^{*}\left(L M, \mathrm{ev}^{*} P_{\mathfrak{u}}\right) \rightarrow \Omega^{*}\left(L M, \mathrm{ev}^{*} \operatorname{ad} P\right) .
\end{aligned}
$$

Definition 6.1 For a differential form $\alpha \in \Omega^{*}(M, \operatorname{ad} P)$, the Wilson loop $\mathcal{W}_{\alpha} \in$ $\Omega^{*}(L M, \mathbb{R})$ is defined as,

$$
\mathcal{W}_{\alpha}=\widehat{f}\left(W_{\alpha}\right)
$$

Proposition 6.2 If $\alpha \in M C$, then $\mathcal{W}_{\alpha} \in \Omega^{*}(L M)$ is a closed form.

Proof Using Stokes' theorem we have

$$
\begin{aligned}
d \mathcal{W}_{\alpha}=d \hat{f}(\mathrm{hol}, 1) & +\sum_{n=1}^{\infty} \hat{f}\left(\mathrm{hol}, d \int_{\Delta^{n}} \alpha(n, 1) \wedge \cdots \wedge \alpha(n, n)\right) \\
& +\sum_{n=1}^{\infty} \hat{f}\left(\mathrm{hol}, \int_{\partial \Delta^{n}} \alpha(n, 1) \wedge \cdots \wedge \alpha(n, n)\right)
\end{aligned}
$$

Since the connection $\nabla$ is flat, $\hat{f}(\mathrm{hol}, 1)=f(\mathrm{hol})$ is constant on the connected components of $L M$ therefore $d \hat{f}(\mathrm{hol})=0$ on $L M$. Actually one can say more, hol $\in \Gamma\left(\operatorname{ev}^{*}\left(P_{G}\right)\right)$ is a flat section. Therefore, by Corollary A.4, we have

$$
\begin{aligned}
d \mathcal{W}_{\alpha}=\sum_{n=1}^{\infty} \hat{f}\left(\mathrm{hol}, \int_{\Delta^{n}} d_{\mathrm{ev}^{*} \nabla}(\alpha(n, 1) \wedge\right. & \cdots \wedge \alpha(n, n))) \\
& +\sum_{n=1}^{\infty} \hat{f}\left(\mathrm{hol}, \int_{\partial \Delta^{n}} \alpha(n, 1) \wedge \cdots \wedge \alpha(n, n)\right) .
\end{aligned}
$$


We analyze different terms separately. For the first part we have

$$
\begin{aligned}
d_{\mathrm{ev}^{*} \nabla}(\alpha(n, 1) \wedge \cdots \wedge & \alpha(n, n)) \\
& =\sum_{i=1}^{n}(-1)^{i} \alpha(n, 1) \wedge \cdots \wedge d_{\mathrm{ev}^{*} \nabla}(\alpha(n, i)) \wedge \cdots \wedge \alpha(n, n) \\
& =\sum_{i=1}^{n}(-1)^{i} \alpha(n, 1) \wedge \cdots \wedge\left(d_{\nabla}(\alpha)\right)(n, i) \wedge \cdots \wedge \alpha(n, n) .
\end{aligned}
$$

Hence, $\sum_{n=1}^{\infty} \int_{\Delta^{n}} \hat{f}\left(\operatorname{hol}, d_{\mathrm{ev}^{*} \nabla}(\alpha(n, 1) \wedge \cdots \wedge \alpha(n, n))\right)=\sum_{n=1}^{\infty} \sum_{i=1}^{n} \hat{f}\left(W_{\alpha, \cdots, d_{\nabla} \alpha, \cdots \alpha}^{n}\right)$.

As for the second part we have $\partial \Delta^{n}=\bigcup_{i=0}^{n} \Delta_{i}^{n-1}$, where,

$$
\Delta_{i}^{n-1}=\left\{\left(t_{0}, t_{1}, \cdots, t_{n}, t_{n+1}\right) \mid 0=t_{0} \leq t_{1} \leq \cdots \leq t_{i}=t_{i+1} \leq \cdots \leq t_{n} \leq t_{n+1}=1\right\},
$$

and then for $n \geq 1$,

$$
\begin{aligned}
& \widehat{f}\left(\mathrm{hol}, \int_{\partial \Delta^{n}} \alpha(n, 1) \wedge \cdots \wedge \alpha(n, n)\right) \\
& =\widehat{f}\left(\mathrm{hol}_{,} \mathrm{Ad}_{\mathrm{hol}}\left(\mathrm{ev}^{*} \alpha\right) \wedge \int_{\Delta^{n-1}} \alpha(n-1,1) \wedge \cdots \wedge \alpha(n-1, n-1)\right) \\
& \quad+\sum_{i=1}^{n-1}(-1)^{i} \widehat{f}\left(\mathrm{hol}, \int_{\Delta^{n-1}} \alpha(n-1,1) \wedge \cdots \wedge(\alpha \wedge \alpha)(n-1, i) \wedge \cdots \wedge \alpha(n-1, n)\right) \\
& \quad+(-1)^{n} \hat{f}\left(\mathrm{hol}, \int_{\Delta^{n-1}} \alpha(n-1,1) \wedge \cdots \wedge \alpha(n-1, n-1) \wedge \mathrm{ev}^{*} \alpha\right) .
\end{aligned}
$$

To calculate the first term in the equality above we have used the fact that the parallel transport of $\operatorname{ad} P$ along a loop $\gamma$ is give by $\operatorname{Ad}_{\text {hol }}$ where hol is understood as the parallel transport in the principal bundle $P$. Now, from the invariance of $f$ and the fact that the components of $\alpha$ have odd degrees, it follows that the first term and the last term cancel each other. Therefore, we have

$$
\begin{aligned}
& \sum_{n=1}^{\infty} \widehat{f}\left(\mathrm{hol}, \int_{\partial \Delta^{n}} \alpha(n, 1) \wedge \cdots \wedge \alpha(n, n)\right) \\
& \quad=\sum_{n=2}^{\infty} \sum_{i=1}^{n-1}(-1)^{i} \hat{f}\left(\mathrm{hol}, \int_{\Delta^{n-1}} \alpha(n-1,1) \wedge \cdots \wedge(\alpha \wedge \alpha)(n-1, i) \wedge \cdots \wedge \alpha(n-1, n-1)\right) \\
& =\sum_{n=1}^{\infty} \sum_{i=1}^{n}(-1)^{i} \hat{f}\left(W_{\alpha, \cdots, \alpha \wedge \alpha, \cdots, \alpha}^{n}\right) .
\end{aligned}
$$


Then, $\quad d \mathcal{W}_{\alpha}=\sum_{n=1}^{\infty} \sum_{i=1}^{n}(-1)^{i} \hat{f}\left(W_{\alpha, \cdots, d_{\nabla} \alpha, \cdots, \alpha}^{n}\right)+\sum_{n=1}^{\infty} \sum_{i=1}^{n}(-1)^{i} \hat{f}\left(W_{\alpha, \cdots, \alpha \wedge \alpha, \cdots, \alpha}^{n}\right)$

$$
=\sum_{n=1}^{\infty} \sum_{i=1}^{n}(-1)^{i} \hat{f}\left(W_{\alpha, \cdots, d_{\nabla} \alpha+\alpha \wedge \alpha, \cdots, \alpha}^{n}\right) .
$$

Note that since $\alpha$ is a sum of forms of odd degrees in $\Omega^{*}\left(M, P_{\mathfrak{u}}\right), \alpha \wedge \alpha=1 / 2[\alpha, \alpha]$. Therefore,

$$
d \mathcal{W}_{\alpha}=\sum_{n=1}^{\infty} \sum_{i=1}^{n}(-1)^{i} \hat{f}\left(W_{\alpha, \cdots, d_{\nabla} \alpha+1 / 2[\alpha, \alpha], \cdots, \alpha}^{n}\right)=0
$$

We now explain how $\mathcal{W}_{\alpha}$ represents an equivariant cohomology class.

Lemma 6.3 For $\alpha \in \mathrm{MC}$, there exists a $\overline{\mathcal{W}}_{\alpha} \in H_{S^{1}}^{*}(L M)$ such that

$$
\mathcal{W}_{\alpha}=\mathfrak{e}^{*}\left(\overline{\mathcal{W}}_{\alpha}\right)
$$

Proof By (5-2) it suffices to show that $\mathcal{W}_{\alpha} \in \Omega^{*}(L M) \stackrel{1 \otimes \text { id }}{\hookrightarrow} \Omega^{*}(L M) \otimes \Omega^{*}\left(S^{\infty}\right)$ is a basic form. Since $\overline{\mathcal{W}}_{\alpha}$ is a closed form, it remains to show that $i_{v} \mathcal{W}_{\alpha}=0$. The fact that $i_{v} \mathcal{W}_{\alpha}=0$ for $v=\frac{\partial}{\partial t}$, the fundamental vector field of the $S^{1}$-action, is obvious since $\mathcal{W}_{\alpha}$ is obtained by pullback of the evaluation maps, $\operatorname{dev}_{t_{k}}(v)=\gamma^{\prime}\left(t_{k}\right)$ and $i_{\gamma^{\prime}\left(t_{k}\right)} i_{\gamma^{\prime}\left(t_{k}\right)}=0$. This shows that $\mathcal{W}_{\alpha}$ determines a cohomology class $\overline{\mathcal{W}}_{\alpha} \in H_{S^{1}}^{*}(L M)$ which satisfies

$$
\mathcal{W}_{\alpha}=\mathfrak{e}^{*}\left(\overline{\mathcal{W}}_{\alpha}\right)
$$

Since the equivariant de Rham cohomology of $L M$ is isomorphic to the singular one, we can think of $\overline{\mathcal{W}}_{\alpha}$ as an element in $H_{S^{1}}^{*}(L M, \mathbb{R})$. For $c \in H_{*}^{S^{1}}(L M)$, define $\Psi_{c} \in \mathcal{O}(\mathrm{MC})$ as

$$
\Psi_{c}(\alpha)=\left\langle c, \overline{\mathcal{W}}_{\alpha}\right\rangle
$$

The following proposition implies that $\Psi_{c}$ descends to $\mathcal{O}(\mathcal{M C})$.

Proposition 6.4 For $c \in H_{*}(L M)$ and $a \in \mathcal{L}_{0}=\Omega^{2 *}(M, \operatorname{ad} P)$,

$$
L_{\xi_{a}} \Psi_{c}=0 \in \mathcal{O}(\mathrm{MC}) .
$$

Proof By Cartan's formula, we have

$$
\left(L_{\xi_{a}} \Psi_{c}\right)(\alpha)=\left(i_{\xi_{a}} d \Psi_{c}+d i_{\xi_{a}} \Psi_{c}\right)(\alpha)=i_{\xi_{a}} d \Psi_{c}(\alpha) .
$$


Let us calculate $d \Psi_{c}(\alpha)(h)$ for $h \in \mathcal{L}_{1}=T_{\alpha} \mathcal{L}_{1}$,

$$
d \Psi_{c}(\alpha)(h)=\left.\frac{d}{d t}\left(\int_{c} \overline{\mathcal{W}}_{\alpha+t h}\right)\right|_{t=0}=\left.\int_{c} \frac{d}{d t}\left(\overline{\mathcal{W}}_{\alpha+t h}\right)\right|_{t=0}
$$

Note that

$$
\left.\frac{d}{d t} \mathcal{W}_{\alpha+t h}\right|_{t=0}=\left.\frac{d}{d t} \sum_{n=1}^{\infty} \widehat{f}\left(W_{\alpha+t h, \cdots, \alpha+t h}^{n}\right)\right|_{t=0}=\sum_{n=1}^{\infty} \sum_{i=1}^{n} \widehat{f}\left(W_{\alpha, \cdots, h, \cdots, \alpha}^{n}\right)
$$

So, in order to show $L_{\xi_{a}} \Psi_{c}(\alpha)=i_{\xi_{a}} d \Psi_{c}(\alpha)=0$ we have to show $\left.\frac{d}{d t} \mathcal{W}_{\alpha+t h}\right|_{t=0}$ is an exact form for $h=\xi_{a}(\alpha)$, or in other words,

$$
\sum_{n=1}^{\infty} \sum_{i=1}^{n} \widehat{f}\left(W_{\alpha, \cdots, \xi_{a}(\alpha), \cdots, \alpha}^{n}\right)
$$

is exact.

Let $Z=\sum_{n=1}^{\infty} \sum_{i=1}^{n}(-1)^{i} \hat{f}\left(W_{\alpha, \cdots, a, \cdots, \alpha}^{n}\right) \in \Omega^{*}(L M, \mathbb{R})$. Then, by a calculation similar to that presentation in the proof of Proposition 6.2 we show that for $a \in \mathcal{L}_{+}$,

$$
\begin{aligned}
& d Z=\sum_{n=1}^{\infty} \sum_{i=1}^{n}(-1)^{2 i}\left(\hat{f}(W_{\alpha, \cdots,}^{n}, \underbrace{d_{\nabla} a}_{i}, \cdots, \alpha)+\hat{f}(W_{\alpha, \cdots,}^{n}, \underbrace{\alpha \wedge a}_{i}, \cdots, \alpha)-\hat{f}(W_{\alpha, \cdots,}^{n}, \underbrace{a \wedge \alpha, \cdots, \alpha}_{i})\right. \\
& +\sum_{n=1}^{\infty} \sum_{i=1}^{n}(-1)^{i}\left[\sum_{j<i}(-1)^{j}\left(\widehat{f}(W_{\alpha, \cdots}^{n} \underbrace{d_{\nabla} \alpha}_{j}, \cdots, \underbrace{, a, \cdots, \alpha}_{i})+\widehat{f}(W_{\alpha, \cdots,}^{n}, \underbrace{\alpha \wedge \alpha}_{j}, \cdots \underbrace{, a, \cdots, \alpha}_{i})\right)\right. \\
& \left.\left.+\sum_{i<j \leq n}(-1)^{j}\left(\hat{f}(W_{\alpha, \cdots,}^{n} \underbrace{, a, \cdots}_{i}, \underbrace{d_{\nabla} \alpha}_{j}, \cdots, \alpha)\right)\right]+\hat{f}\left(W_{\alpha, \cdots, \underbrace{n}_{i}, a, \cdots, \underbrace{\alpha \wedge \alpha}_{j}, \cdots, \alpha}^{n}\right)\right)
\end{aligned}
$$

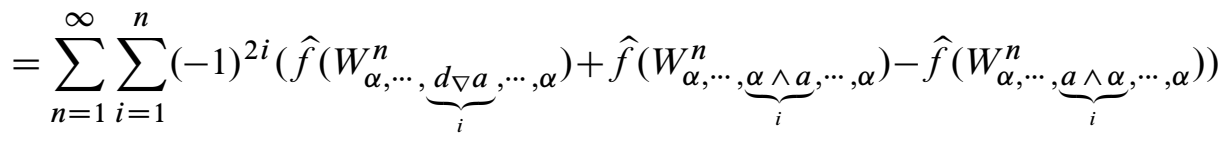

$$
\begin{aligned}
& +\sum_{n=1}^{\infty} \sum_{i=1}^{n}(-1)^{i}\left(\sum_{j<i}(-1)^{j} \hat{f}(W_{\alpha, \cdots,}^{n} \underbrace{d_{\nabla} \alpha+1 / 2[\alpha, \alpha]}_{j}, \cdots, \underbrace{, a, \cdots, \alpha}_{i})\right. \\
& \left.+\sum_{i<j \leq n}(-1)^{j} \hat{f}(W_{\alpha, \cdots,}^{n} \underbrace{, a, \cdots}_{i}, \underbrace{d_{\nabla} \alpha+1 / 2[\alpha, \alpha]}_{j}, \cdots, \alpha)\right) \text {. }
\end{aligned}
$$

Algebraic $\&$ Geometric Topology, Volume 7 (2007) 
As $d_{\nabla} \alpha+\frac{1}{2}[\alpha, \alpha]=0$ for $\alpha \in \mathrm{MC}$, therefore,

$$
\begin{aligned}
d Z & =\sum_{n=1}^{\infty} \sum_{i=1}^{n} \hat{f}\left(W_{\alpha, \cdots, d_{\nabla} a+[\alpha, a], \cdots, \alpha}^{n}\right) \\
& =\sum_{n=1}^{\infty} \sum_{i=1}^{n} \hat{f}\left(W_{\alpha, \cdots, \xi_{a}(\alpha), \cdots, \alpha}^{n}\right) \\
& =\left.\frac{d}{d t} \mathcal{W}_{\alpha+t \xi_{a}(\alpha)}\right|_{t=0} .
\end{aligned}
$$

This proves the claim.

By Proposition 6.4, $\Psi_{a}: M C \rightarrow \mathbb{R}$ is constant along the orbits of $\mathcal{G}$.

Definition 6.5 The generalized holonomy map $\Psi: H_{2 *}^{S^{1}}(L M) \rightarrow \mathcal{O}(\mathcal{M C})$ is the map $a \mapsto \Psi_{a}$, where

$$
\Psi_{a}(\alpha)=\left\langle a, \overline{\mathcal{W}}_{\alpha}\right\rangle
$$

\section{Hamiltonian vectors field and Poisson bracket}

Let $\Psi_{a}$, and $\Psi_{b}$ denote the holonomy functions associated to the equivariant homology classes $a, b \in H_{2 *}^{S^{1}}(L M)$, and let $X_{a}$ and $X_{b}$ denote their corresponding Hamiltonian vector fields. We calculate the Poisson bracket of holonomy functions by evaluating the symplectic form on their corresponding Hamiltonian vector fields. Note that the Zarisky tangent space of $\mathrm{MC} / \mathcal{G}$ at a class represented by $\alpha$ can be identified with the cohomology whose differential is $\nabla+\alpha \wedge \cdot$ or in other words ad $P$ is equipped with the flat connection $\nabla+\alpha \wedge \cdot$. The later is a differential as $\alpha$ satisfies the MaurerCartan equation. We denote the cohomology of this differential by $H^{*}\left(M, \operatorname{ad} P_{\alpha}\right)$, and similarly the corresponding vector bundle homology by $H_{*}\left(M, \operatorname{ad} P_{\alpha}\right)$.

We borrow the following lemma from [17] to obtain a formula for the Hamiltonian vector fields.

Lemma 7.1 The following diagram is commutative:

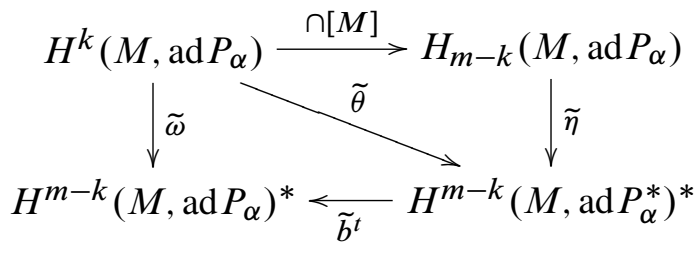

Algebraic $8 \mathcal{G}$ Geometric Topology, Volume 7 (2007) 
where

(i) $[M] \in H_{d}(M)$ is the fundamental class of $M$

(ii) $\widetilde{\omega}$ is induced by $\omega: H^{k}\left(M, \operatorname{ad} P_{\alpha}\right) \otimes H^{m-k}\left(M, \operatorname{ad} P_{\alpha}\right) \stackrel{\int_{M}\langle\cdot, \cdot\rangle}{\longrightarrow} \mathbb{R}$

(iii) $\tilde{\theta}$ is induced by $\theta: H^{k}\left(M, \operatorname{ad} P_{\alpha}\right) \otimes H^{m-k}\left(M, \operatorname{ad} P_{\alpha}^{*}\right) \stackrel{\left(\int_{M} \cdot \wedge \cdot\right) \otimes \mathrm{eval}}{\longrightarrow} \mathbb{R}$

(iv) $\tilde{\eta}$ is induced by $\eta: H_{m-k}\left(M, \operatorname{ad} P_{\alpha}\right) \otimes H^{m-k}\left(M\right.$, ad $\left.P_{\alpha}^{*}\right) \stackrel{\text { eval }}{\longrightarrow} \mathbb{R}$

(v) $\tilde{b}^{t}$ is the transpose of the map $\tilde{b}: H^{m-k}\left(M, \operatorname{ad} P_{\alpha}\right) \rightarrow H^{m-k}\left(M, \operatorname{ad} P_{\alpha}^{*}\right)$ induced by $\langle\cdot, \cdot\rangle: \mathfrak{g} \times \mathfrak{g} \rightarrow \mathbb{R}$.

Here, $\operatorname{ad} P_{\alpha}^{*}$ is the bundle associated to the representation $\operatorname{Ad}^{*}: G \rightarrow \operatorname{Aut}\left(\mathfrak{g}^{*}\right)$ induced by the conjugation action, with the differential induced by $\nabla+\alpha \wedge \cdot$.

Lemma 7.2 The Hamiltonian vector field $X_{a}(\alpha) \in H^{*}\left(M, \operatorname{ad} P_{\alpha}\right)$, for $a \in H_{*}(L M)$, satisfies the equation

$$
P D\left(X_{a}(\alpha)\right)=\mathrm{ev}_{*}\left(\mathfrak{m}_{*} a \cap \widehat{F}\left(W_{\alpha}\right)\right)=\sum_{k=0}^{\infty} \mathrm{ev}_{*}\left(\mathfrak{m}_{*} a \cap \widehat{F}\left(W_{\alpha}^{k}\right)\right),
$$

where $P D$ denotes the Poincaré duality map $H^{*}\left(M, \operatorname{ad} P_{\alpha}\right) \stackrel{\cdot \cap[M]}{\longrightarrow} H_{*}\left(M, \operatorname{ad} P_{\alpha}\right)$.

Proof For the Hamiltonian vector field $X_{a}(\alpha)$ and $h \in T_{\alpha} \mathcal{M C}$,

$$
\omega\left(X_{a}(\alpha), h\right)=\left.\frac{d}{d t} \Psi_{a}(\alpha+t h)\right|_{t=0}=\left.\frac{d}{d t}\left\langle a, \overline{\mathcal{W}}_{\alpha+t h}\right\rangle\right|_{t=0}=\left.\left\langle a, \frac{d}{d t}, \overline{\mathcal{W}}_{\alpha+t h}\right\rangle\right|_{t=0}
$$

Note that $\overline{\mathcal{W}}_{\alpha+t h}$ is represented by the differential form,

$$
\mathcal{W}_{\alpha+t h}=\sum_{k=0}^{\infty} \hat{f}\left(W_{\alpha+t h, \cdots, \alpha+t h}^{k}\right) .
$$

Because of the flatness of $\nabla, d \hat{f}($ hol $)=0$, therefore,

$$
\mathfrak{e}^{*}\left(\left.\frac{d}{d t} \overline{\mathcal{W}}_{\alpha+t h}\right|_{t=0}\right)=\sum_{k=1}^{\infty} \sum_{i=1}^{n} \hat{f}\left(W_{\alpha \cdots \underbrace{k, h, \cdots, \alpha}_{i}}^{k}\right),
$$

which by [14, Theorem 2.1] is

$$
\sum_{k=1}^{\infty} \Delta^{*} \hat{f}\left(\mathrm{hol}, V_{\alpha}^{k} \wedge \mathrm{ev}^{*} h\right)=\sum_{k=0}^{\infty} \mathfrak{e}^{*} \mathfrak{m}^{*} \hat{f}\left(\mathrm{hol}, V_{\alpha}^{k} \wedge \mathrm{ev}^{*} h\right) .
$$

Algebraic $8 \mathcal{G}$ Geometric Topology, Volume 7 (2007) 
Hence by (3-2) and (3-1),

$$
\begin{aligned}
\omega\left(X_{a}(\alpha), h\right) & =\left\langle a, \mathfrak{m}^{*} \sum_{k=0}^{\infty} \hat{f}\left(\mathrm{hol}, V_{\alpha}^{k} \wedge \mathrm{ev}^{*} h\right)\right\rangle \\
& =\left\langle\mathfrak{m}_{*} a, \sum_{k=0}^{\infty} \hat{f}\left(\mathrm{hol}, V_{\alpha}^{k} \wedge \mathrm{ev}^{*} h\right)\right\rangle \\
& =\left\langle\mathfrak{m}_{*} a \cap \sum_{k=0}^{\infty} \widehat{F}\left(W_{\alpha}^{k}\right), \mathrm{ev}^{*} h\right\rangle \\
& =\left\langle\operatorname{ev}_{*}\left(\mathfrak{m}_{*} a \cap \sum_{k=0}^{\infty} \hat{F}\left(W_{\alpha}^{k}\right)\right), h\right\rangle .
\end{aligned}
$$

Therefore, by Lemma 7.1,

$$
\begin{aligned}
\operatorname{ev}_{*}\left(\mathfrak{m}_{*} a \cap \sum_{k=0}^{\infty} \hat{F}\left(W_{\alpha}^{k}\right)\right) & =\eta^{-1} \circ\left(\tilde{b}^{t}\right)^{-1}\left(d_{\alpha} \Psi_{a}\right) \\
& =\tilde{\omega}^{-1}\left(d_{\alpha} \Psi_{a}\right) \cap[M] \\
& =X_{a}(\alpha) \cap[M] .
\end{aligned}
$$

The following lemma is a version of the multiplicative property of the formal power series parallel transport $[7 ; 8 ; 22]$. See $(3-4)$ to recall the definition of the $*$ product.

Lemma 7.3 Let $\varepsilon: L M \times_{M} L M \rightarrow L M$ be the concatenation map. Then

$$
\varepsilon^{*}\left(W_{\alpha}\right)=\operatorname{pr}_{1}^{*} W_{\alpha} * \operatorname{pr}_{2}^{*} W_{\alpha},
$$

where $\mathrm{pr}_{1}$ and $\mathrm{pr}_{2}$ are the projections on the first and the second factors,

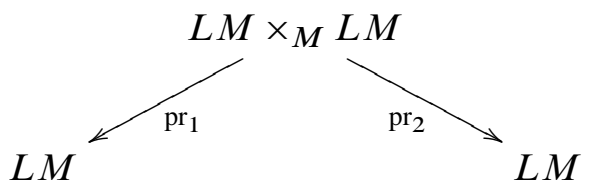

Proof The proof of Proposition 1.5.1 in [7] can be adopted to prove that for two loops $\gamma_{1}$ and $\gamma_{2}$ with identical marked point,

$$
V_{\alpha}^{n}\left(\gamma_{1} \circ \gamma_{2}\right)=\sum_{i+j=n} \operatorname{Ad}_{\mathrm{hol}\left(\gamma_{2}\right)} V_{\alpha}^{i}\left(\gamma_{1}\right) \wedge V_{\alpha}^{j}\left(\gamma_{2}\right)
$$

Also $\operatorname{hol}\left(\gamma_{1} \circ \gamma_{2}\right)=\operatorname{hol}\left(\gamma_{2}\right) \circ \operatorname{hol}\left(\gamma_{1}\right)$. To see the identity (7-1) more clearly, recall that the parallel transport in $\operatorname{ad} P$ along $\gamma_{2}$ is given by $\operatorname{Ad}_{\text {hol }}\left(\gamma_{2}\right)$, and that in computing the differential form $V_{\alpha}^{n}\left(\gamma_{1} \circ \gamma_{2}\right)$ (see Section 6) the parallel transport $T_{i}$ along $\gamma_{1} \circ \gamma_{2}$ 
acts on all the pullbacks $\mathrm{ev}_{t_{i}}^{*} \alpha$. Split the terms $\alpha(n, i)$ into two collections according to whether they are obtained by the pullback with respect to $\mathrm{ev}_{t}$ for $0 \leq t \leq 1 / 2$ or $1 / 2 \leq t \leq 1$. Then, note that one needs to apply an extra action of $\operatorname{Ad}_{\text {hol }\left(\gamma_{2}\right)}$ on the first collection to account for the parallel transport along the second half of $\gamma_{1} \circ \gamma_{2}$, that is $\gamma_{2}$.

Recall Definition 6.5 wherein the generalized holonomy map $\Psi: H_{2 *}^{S^{1}}(L M) \rightarrow \mathcal{O}(\mathcal{M C})$ is described as the map $a \mapsto \Psi_{a}$, where, $\Psi_{a}(\alpha)=\left\langle a, \overline{\mathcal{W}}_{\alpha}\right\rangle$. For a version of the following theorem in the BRST setup, see Cattaneo, Fröhlich and Pedrini [3].

Theorem 7.4 For $G=\operatorname{GL}(n, \mathbb{C})$ or $\operatorname{GL}(n, \mathbb{R})$, the generalized holonomy map

$$
\Psi:\left(H_{2 *}^{S^{1}}(L M),[\cdot, \cdot]\right) \rightarrow(\mathcal{O}(\mathcal{M C}),\{\cdot, \cdot\}),
$$

is a map of Lie algebras.

Proof Suppose that $G=\mathrm{GL}(n, \mathbb{C})$, it is exactly the same argument for $G=\mathrm{GL}(n, \mathbb{R})$. We prove that for $a, b \in H_{2 *}^{S^{1}}(L M)$ and $\alpha \in \mathcal{M C}$,

$$
\Psi_{[a, b]}(\alpha)=\left\{\Psi_{a}, \Psi_{b}\right\}(\alpha)=\omega\left(X_{a}(\alpha), X_{b}(\alpha)\right) .
$$

The left hand side is

$$
\begin{aligned}
\Psi_{[a, b]}(\alpha) & =\Psi_{\left(\mathfrak{e}_{*}\left(\mathfrak{m}_{*} a \bullet \mathfrak{m}_{*} b\right)\right.}(\alpha) \\
& =\left\langle\mathfrak{e}_{*}\left(\mathfrak{m}_{*} a \bullet \mathfrak{m}_{*} b\right), \overline{\mathcal{W}}_{\alpha}\right\rangle \\
& =\left\langle\mathfrak{m}_{*} a \bullet \mathfrak{m}_{*} b, \mathfrak{e}^{*}\left(\overline{\mathcal{W}}_{\alpha}\right)\right\rangle \\
& =\left\langle\mathfrak{m}_{*} a \bullet \mathfrak{m}_{*} b, \mathcal{W}_{\alpha}\right\rangle \\
& =\left\langle\varepsilon_{*} \circ \tau_{*}\left(\mathfrak{m}_{*} a \times \mathfrak{m}_{*} b\right), \hat{\operatorname{tr}}\left(W_{\alpha}\right)\right\rangle \\
& =\left\langle\mathfrak{m}_{*} a \times \mathfrak{m}_{*} b, \hat{\operatorname{tr}}\left(\tau^{*} \circ \varepsilon^{*}\left(W_{\alpha}\right)\right)\right\rangle
\end{aligned}
$$

which using Lemma 7.3 equals

$$
\int_{\mathfrak{m}_{*} a \times \mathfrak{m}_{*} b}(\mathrm{ev} \times \mathrm{ev})^{*}(U) \wedge \widehat{\operatorname{tr}}\left(\operatorname{pr}_{1}^{*} W_{\alpha} * \operatorname{pr}_{2}^{*} W_{\alpha}\right),
$$

where $U \in \Omega^{*}(M \times M)$ is a differential form supported in a neighborhood of the diagonal realizing the Thom isomorphism. Now, by Lemma 7.2,

$$
\begin{aligned}
\left\{\Psi_{a}, \Psi_{b}\right\}(\alpha) & =\omega\left(X_{a}(\alpha), X_{b}(\alpha)\right) \\
& =\int_{M} \operatorname{tr}\left(X_{a}(\alpha) \wedge X_{b}(\alpha)\right) \\
& =\int_{M} \operatorname{tr}\left(P D ^ { - 1 } \left(\mathrm{ev}_{*}\left(\mathfrak{m}_{*} a \cap \widehat{F}\left(W_{\alpha}\right)\right) \wedge P D^{-1}\left(\mathrm{ev}_{*}\left(\mathfrak{m}_{*} a \cap \widehat{F}\left(W_{\alpha}\right)\right)\right) .\right.\right.
\end{aligned}
$$

Algebraic $8 \mathcal{G}$ Geometric Topology, Volume 7 (2007) 
Using Remark 3.4 and the inclusion $\Omega^{*}\left(L M, \mathrm{ev}^{*} P_{G}\right) \hookrightarrow \Omega^{*}\left(L M, \mathrm{ev}^{*}\right.$ ad $\left.P\right)$ induced by $\operatorname{GL}(n, \mathbb{C}) \hookrightarrow \mathfrak{g} l(n, \mathbb{C})$, and by Corollary 3.2 ,

$$
\widehat{F}\left(W_{\alpha}\right)=\operatorname{hol} \cdot \Pi V_{\alpha} \in \Omega^{*}\left(L M, \mathrm{ev}^{*} \operatorname{ad} P\right) .
$$

Thus, the above Poisson bracket equals

$$
\begin{aligned}
& \int_{\operatorname{tr}\left(\mathrm{ev}_{*}\left(\mathfrak{m}_{*} a \cap \hat{F}\left(W_{\alpha}\right)\right) \times \mathrm{ev}_{*}\left(\mathfrak{m}_{*} a \cap \widehat{F}\left(W_{\alpha}\right)\right)\right)} U \\
& =\int_{\left(\mathfrak{m}_{*} a \times \mathfrak{m}_{*} b\right) \cap \operatorname{tr}\left(\hat{F}\left(W_{\alpha}\right) \times \hat{F}\left(W_{\alpha}\right)\right)}(\mathrm{ev} \times \mathrm{ev})^{*} U \\
& =\int_{\mathfrak{m}_{*} a \times \mathfrak{m}_{*} b}(\mathrm{ev} \times \mathrm{ev})^{*} U \wedge \operatorname{tr}\left(\operatorname{pr}_{1}^{*} \hat{F}\left(W_{\alpha}\right) \wedge \operatorname{pr}_{2}^{*} \hat{F}\left(W_{\alpha}\right)\right)
\end{aligned}
$$

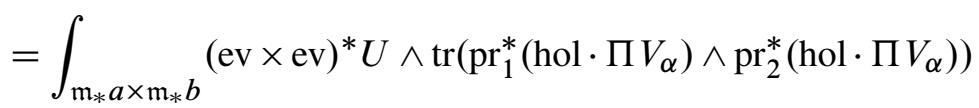

$$
\begin{aligned}
& =\int_{\mathfrak{m}_{*} a \times \mathfrak{m}_{*} b}(\mathrm{ev} \times \mathrm{ev})^{*} U \wedge \operatorname{tr}\left(\left(\operatorname{pr}_{1}^{*} \mathrm{hol} \cdot \mathrm{pr}_{1}^{*} \Pi V_{\alpha}\right) \wedge\left(\mathrm{pr}_{2}^{*} \mathrm{hol} \cdot \mathrm{pr}_{2}^{*} \Pi V_{\alpha}\right)\right) .
\end{aligned}
$$

But, it follows immediately from Remark 3.4 and line (3-3) that

$$
\operatorname{tr}\left(\left(\operatorname{pr}_{1}^{*} \text { hol } \cdot \operatorname{pr}_{1}^{*} \Pi V_{\alpha}\right) \wedge\left(\operatorname{pr}_{2}^{*} \text { hol } \cdot \operatorname{pr}_{2}^{*} \Pi V_{\alpha}\right)\right)=\widehat{\operatorname{tr}}\left(\operatorname{pr}_{1}^{*} W_{\alpha} * \operatorname{pr}_{2}^{*} W_{\alpha}\right) .
$$

This proves the theorem.

\section{String bracket for unoriented strings}

In this section we calculate the Poisson bracket for the generalized holonomy associated to a principal $G$-bundle equipped with a flat connection, where $G$ is one of the proper reductive subgroups of $\mathrm{GL}(n, \mathbb{C})$ such as $\mathrm{O}(n, \mathbb{R}), \mathrm{O}(n, \mathbb{C}), \mathrm{U}(n), \operatorname{SL}(n, \mathbb{R})$, or $\operatorname{SL}(n, \mathbb{C})$. Let $i: L M \rightarrow L M$ be the involution which reverses the orientation of a loop. We continue to denote the induced map on the homology $H_{*}(L M)$ and the equivariant homology $H_{*}^{S^{1}}(L M)$ by $a \mapsto i(a)$. We use $i^{*}$ for the induced map on the cohomologies. It is a direct check that the latter maps commute with the maps $\mathfrak{e}_{*}$ and $\mathfrak{m}_{*}$ introduced in Section 2.

Theorem 8.1 For $G=\mathrm{O}(p, q), \mathrm{O}(n, \mathbb{C}), \mathrm{U}(p, q), \operatorname{Sp}(n, \mathbb{R})$ and $\operatorname{Sp}(p, q)$,

$$
\left\{\Psi_{a}, \Psi_{b}\right\}=\frac{1}{2}\left(\Psi_{[a, b]}-\Psi_{[a, i(b)]}\right),
$$

where $a, b \in H_{2 *}^{S^{1}}(L M)$. 
Proof Consider

$$
V_{\alpha}^{t}=\sum_{n=0}^{\infty}(-1)^{n} \int_{\Delta^{n}} \alpha(n, n) \wedge \cdots \wedge \alpha(n, 1) .
$$

Notice that first term of the above summation is 1 (compare (6-1)). In a manner similar to the proof of Theorem 7.4 ,

$$
\left.\left\{\Psi_{a}, \Psi_{b}\right\}(\alpha)=\int_{\mathfrak{m}_{*} a \times \mathfrak{m}_{*} b}(\mathrm{ev} \times \mathrm{ev})^{*} U \wedge \operatorname{tr}\left(\operatorname{pr}_{1}^{*} \hat{F}\left(W_{\alpha}\right) \wedge \operatorname{pr}_{2}^{*} \hat{F}\left(W_{\alpha}\right)\right)\right),
$$

By Corollary 3.3,

$$
\widehat{F}\left(W_{\alpha}\right)=\frac{1}{2}\left(\mathrm{hol} \cdot \Pi V_{\alpha}-\Pi V_{\alpha}^{t} \cdot \operatorname{hol}^{-1}\right) \in \Omega^{*}\left(L M, \mathrm{ev}^{*} \mathrm{ad} P\right),
$$

and all the terms above make sense using the fact $G$ is one of the subgroups listed in the theorem. Since for every $g \in G, \operatorname{Re} \operatorname{tr}(A)=\operatorname{Re} \operatorname{tr}\left(A^{-1}\right)$ it follows,

$$
\begin{aligned}
\left\{\Psi_{a}, \Psi_{b}\right\}(\alpha) & \\
= & \frac{1}{2} \int_{\mathfrak{m}_{*} a \times \mathfrak{m}_{*} b}(\mathrm{ev} \times \mathrm{ev})^{*} U \wedge \operatorname{tr}\left(\operatorname{pr}_{1}^{*}(\mathrm{hol}) \operatorname{pr}_{1}^{*}\left(\Pi V_{\alpha}\right) \wedge \operatorname{pr}_{2}^{*}(\mathrm{hol}) \operatorname{pr}_{2}^{*}\left(\Pi V_{\alpha}\right)\right) \\
& -\frac{1}{2} \int_{\mathfrak{m}_{*} a \times \mathfrak{m}_{*} b}(\mathrm{ev} \times \mathrm{ev})^{*} U \wedge \operatorname{tr}\left(\operatorname{pr}_{1}^{*}(\mathrm{hol}) \operatorname{pr}_{1}^{*}\left(\Pi V_{\alpha}\right) \wedge \operatorname{pr}_{2}^{*}\left(\Pi V_{\alpha}^{t}\right) \operatorname{pr}_{2}^{*}\left(\mathrm{hol}^{-1}\right)\right) .
\end{aligned}
$$

Also, as in the proof of Theorem 7.4,

$$
\begin{aligned}
\frac{1}{2}\left(\Psi_{[a, b]}(\alpha)-\Psi_{[a, i(b)]}(\alpha)\right)= & \frac{1}{2} \int_{\mathfrak{m}_{*} a \times \mathfrak{m}_{*} b}(\mathrm{ev} \times \mathrm{ev})^{*}(U) \wedge \operatorname{tr}\left(\operatorname{pr}_{1}^{*} W_{\alpha} * \operatorname{pr}_{2}^{*} W_{\alpha}\right) \\
& -\frac{1}{2} \int_{\mathfrak{m}_{*} a \times \mathfrak{m}_{*} i(b)}(\mathrm{ev} \times \mathrm{ev})^{*}(U) \wedge \operatorname{tr}\left(\operatorname{pr}_{1}^{*} W_{\alpha} * \operatorname{pr}_{2}^{*} W_{\alpha}\right) .
\end{aligned}
$$

Similarly to the proof of Theorem 7.4,

$$
\begin{aligned}
\int_{\mathfrak{m}_{*} a \times \mathfrak{m}_{*} b} \operatorname{tr}\left(\operatorname{pr}_{1}^{*}(\mathrm{hol}) \operatorname{pr}_{1}^{*} \Pi V_{\alpha} \wedge \operatorname{pr}_{2}^{*}(\mathrm{hol}) \operatorname{pr}_{2}^{*} \Pi V_{\alpha}\right) \\
\quad=\int_{\mathfrak{m}_{*} a \times \mathfrak{m}_{*} b}(\mathrm{ev} \times \mathrm{ev})^{*}(U) \wedge \hat{\operatorname{tr}}\left(\mathrm{pr}_{1}^{*} W_{\alpha} * \mathrm{pr}_{2}^{*} W_{\alpha}\right) .
\end{aligned}
$$

So, we only have to show that

$$
\begin{aligned}
\int_{\mathfrak{m}_{*} a \times \mathfrak{m}_{*} b} \operatorname{tr}\left(\operatorname{pr}_{1}^{*}(\mathrm{hol}) \operatorname{pr}_{1}^{*} \Pi V_{\alpha} \wedge \operatorname{pr}_{2}^{*} \Pi V_{\alpha}^{t} \operatorname{pr}_{2}^{*}\left(\mathrm{hol}^{-1}\right)\right) \\
=\int_{\mathfrak{m}_{*} a \times \mathfrak{m}_{*} i(b)}(\mathrm{ev} \times \mathrm{ev})^{*}(U) \wedge \widehat{\operatorname{tr}}\left(\operatorname{pr}_{1}^{*} W_{\alpha} * \operatorname{pr}_{2}^{*} W_{\alpha}\right) .
\end{aligned}
$$

Algebraic $8 \mathcal{G}$ Geometric Topology, Volume 7 (2007) 
Note that $\mathrm{ev} \circ i=\mathrm{ev}$, and therefore $(\mathrm{ev} \times \mathrm{ev})^{*} U=(\mathrm{id} \times i)^{*}(\mathrm{ev} \times \mathrm{ev})^{*} U$. Also,

$$
\begin{aligned}
\int_{\mathfrak{m}_{*} a \times \mathfrak{m}_{*} i(b)}(\mathrm{ev} \times \mathrm{ev})^{*} U \wedge \widehat{\operatorname{tr}}\left(\mathrm{pr}_{1}^{*} W_{\alpha} * \operatorname{pr}_{2}^{*} W_{\alpha}\right) \\
\quad=\int_{\mathfrak{m}_{*} a \times \mathfrak{m}_{*} b}(\mathrm{id} \times i)^{*}\left((\mathrm{ev} \times \mathrm{ev})^{*}(U) \wedge \widehat{\operatorname{tr}}\left(\mathrm{pr}_{1}^{*} W_{\alpha} * \operatorname{pr}_{2}^{*} W_{\alpha}\right)\right) \\
\quad=\int_{\mathfrak{m}_{*} a \times \mathfrak{m}_{*} b}(\mathrm{ev} \times \mathrm{ev})^{*} U \wedge \widehat{\operatorname{tr}}\left(\operatorname{pr}_{1}^{*} W_{\alpha} * \operatorname{pr}_{2}^{*}\left(i^{*}\left(W_{\alpha}\right)\right)\right.
\end{aligned}
$$

Note ${ }^{3}$ that the pullback $i^{*}\left(W_{\alpha}\right)=\left(\mathrm{hol}^{-1}, \mathrm{Ad}_{\mathrm{hol}^{-1}} V_{\alpha}^{t}\right)$, and therefore by Remark 3.4 and equation (3-3),

$$
\begin{aligned}
\widehat{\operatorname{tr}}\left(\operatorname{pr}_{1}^{*} W_{\alpha} * \operatorname{pr}_{2}^{*}\left(i^{*}\left(W_{\alpha}\right)\right)\right) & =\widehat{\operatorname{tr}}\left(\operatorname{pr}_{1}^{*}\left(\text { hol, } V_{\alpha}\right) * \operatorname{pr}_{2}^{*}\left(\operatorname{hol}^{-1}, \operatorname{Ad}_{\mathrm{hol}^{-1}} V^{t}\right)\right) \\
& =\operatorname{tr}\left(\operatorname{pr}_{1}^{*}(\mathrm{hol}) \cdot \operatorname{pr}_{1}^{*} \Pi V_{\alpha} \wedge \operatorname{pr}_{2}^{*} \Pi V_{\alpha}^{t} \cdot \operatorname{pr}_{2}^{*}\left(\operatorname{hol}^{-1}\right)\right)
\end{aligned}
$$

This verifies equation (8-1).

For a homology class $a \in H_{*}^{S^{1}}(L M)$, let $\bar{a}=a+i(a)$ which could be thought of as a homology class of unoriented loops.

Lemma 8.2 For $a \in H_{*}^{S^{1}}(L M), a \mapsto i(a)$ is a Lie algebra map.

Proof For $a, b \in H_{*}(L M), i(a) \bullet i(b)=\varepsilon \circ \tau(i(a) \times i(b))=\varepsilon \circ(i, i) \circ \tau(a \times b)$ as the Thom collapsing map commutes with the orientation reversing map. Also,

$$
\varepsilon \circ(i, i) \circ \tau(a \times b)=i \circ \varepsilon \circ \tau(b \times a)=(-1)^{|a||b|} b \bullet a .
$$

As for the Lie bracket,

$$
\begin{aligned}
{[i(a), i(b)] } & =(-1)^{|a|} \mathfrak{e}_{*}\left(\mathfrak{m}_{*}(i(a)) \bullet \mathfrak{m}_{*}(i(b))\right) \\
& =(-1)^{|a|}(-1)^{|a||b|} \mathfrak{e}_{*}\left(i\left(\mathfrak{m}_{*} b \bullet \mathfrak{m}_{*} a\right)\right) \\
& =(-1)^{|a|}(-1)^{|a||b|} i\left(\mathfrak{e}_{*}\left(\mathfrak{m}_{*} b \bullet \mathfrak{m}_{*} a\right)\right) \\
& =(-1)^{|a|+|a||b|+|b|} i([b, a]) \\
& =(-1)^{|a|+|a||b|+|b|}(-1)^{|b|+|a|+|a||b|} i([a, b]) \\
& =i([a, b]) .
\end{aligned}
$$

\footnotetext{
${ }^{3}$ We remind the reader that in our convention $\operatorname{Ad}_{g}(h)=g^{-1} h g$.
} 
Then, it follows from the lemma above that

$$
\begin{aligned}
{[\bar{a}, \bar{b}] } & =[a, b]+[i(a), i(b)]+[i(a), b]+[a, i(b)] \\
& =[a, b]+i([a, b])+i([a, i(b)])+[a, i(b)] \\
& =\overline{[a, b]}+\overline{[a, i(b)]} \\
& =\overline{[a, b]}+\overline{[a, i(b)]} .
\end{aligned}
$$

This motivates a new Lie bracket on $H_{*}^{S^{1}}(L M)$ defined by

$$
[a, b]_{1}=\frac{1}{2} \overline{[\bar{a}, \bar{b}]}=\frac{1}{2}([a, b]+[a, i(b)]) .
$$

Now, using the above observation, Theorem 8.1 can be reformulated as follows:

Theorem 8.3 For $G=\mathrm{O}(p, q), \mathrm{O}(n, \mathbb{C}), \mathrm{U}(p, q), \operatorname{Sp}(n, \mathbb{R})$ and $\operatorname{Sp}(p, q)$,

$$
\left\{\Psi_{a}, \Psi_{b}\right\}=\Psi_{[a, b]_{1}} .
$$

In other words, the map $H_{2 *}^{S^{1}}(L M) \rightarrow \mathcal{O}(\mathcal{M C})$, defined by $a \mapsto \Psi_{a}$, is a map of Lie algebras.

\section{The 2-dimensional case}

In this section we will show how our construction relates to the original construction of Goldman's in $[16 ; 17]$ where he studied the space of flat connection from a representation theory viewpoint.

Let $M$ be a surface with fundamental group $\pi=\pi_{1}(M)$. Then the representation variety $\operatorname{Rep}(\pi, G)$, as a set, is in a one-to-one correspondence with the isomorphism classes of flat $G$-bundles over $M$ (see Proposition A.1). It is known that $\operatorname{Rep}(M, \pi)$ is a symplectic space $[16 ; 1]$. A connected component of $\operatorname{Rep}(\pi, G)$ determines an isomorphism class of principal bundles $G \rightarrow P \rightarrow M$, and the elements of the of each connected component are in a one-to-one correspondence with the flat connections on the corresponding bundle modulo the gauge group.

Let us fix a $G$-bundle $P$, or equivalently, a connected component of $\operatorname{Rep}(\pi, G)$. Then the space of all connections on $G \rightarrow P \rightarrow M$ is an affine space modeled over the vector space $\Omega^{1}(M, \operatorname{ad} P)$. In particular, for a fixed connection $\nabla_{0}$, the map $\alpha \mapsto \nabla_{0}+\alpha$ form $\Omega^{1}$ to the space of all connections is a bijection. Assuming that $\nabla_{0}$ is a flat connection, this map gives a one-to-one correspondence between the set of those 1forms which satisfy the Maurer-Cartan equation and that of all flat connection. In fact, the above map induces a bijection between the equivalence classes of the solution of 
the Maurer-Cartan equation in $\Omega^{1}(M$, ad $P)$, denoted by $\mathcal{M C}$, and the space of flat connections modulo gauge equivalence. In fact, if $(M, \omega)$ is a symplectic manifold of dimension $m=2 d$ for which Hard Lefschetz theorem holds, then the moduli space of flat connections on $P$ may be viewed as a symplectic substack of $\mathcal{M C}$ [13, Section 1.7]. More specifically, the map $\nabla_{0}+\alpha \mapsto 1 / 2\left(\alpha+\alpha \wedge \omega^{d-1}\right)$ identifies the moduli space of flat connections with a symplectic substack of $\mathcal{M C}$ (compare Karshon [21]).

Now, assume $M$ be an orientable manifold of dimension 2. Therefore, for $i \geq 3$, we have $\Omega^{i}(M, \operatorname{ad} P)=0$. We therefore have $\mathcal{M C}_{1}=\mathcal{M C}$. Moreover the symplectic form considered by Goldman for the representation variety corresponds to the one consider in Section $4[16 ; 1]$.

Therefore to make our point it only remains to show how the restriction of the map $\Psi: H_{2 *}^{S^{1}}(L M) \rightarrow \mathcal{O}(\mathcal{M C})$ to the equivariant 0 -th homology $H_{0}^{S^{1}}(L M)$ is precisely the map discovered by Goldman in [17]. First thing to recall is that $H_{0}^{S^{1}}(L M)$ equals $\mathbb{R} \hat{\pi}$, the vector space generated by the set of all conjugacy classes of the fundamental group $\pi_{1}(M)$. Now, for $a \in H_{0}^{S^{1}}(L M)$ look at $\Psi_{a} \in \mathcal{O}(\mathcal{M C})$, where $\Psi_{a}(\alpha)=$ $\left\langle a, \overline{\mathcal{W}}_{\alpha}\right\rangle$. Recall $\mathcal{W}_{\alpha}=\hat{f}\left(W_{\alpha}\right), W_{\alpha}=\left(\mathrm{hol}, V_{\alpha}\right)$, and $V_{\alpha}=\sum_{n=0}^{\infty} V_{\alpha}^{n}$, where $V_{\alpha}^{n}=$ $V_{\alpha, \cdots, \alpha}^{n}$. Since $\alpha$ has to be a $1-$ form, the discussion of Appendix B on iterated integrals says that for a loop in $\gamma \in L M, \Psi_{a}(\gamma)=\left\langle\gamma, \overline{\mathcal{W}}_{\alpha}\right\rangle$ is nothing but the invariant function $f$ applied to the holonomy of the connection $\nabla_{0}+\alpha$ along the loop $\gamma$. Notice that via the one-to-one correspondence of Proposition A.1, this is exactly the function $f_{[\gamma]}$ on $\operatorname{Hom}(\pi, G) / G$ where $[\gamma] \in \hat{\pi}$ is the free homotopy class determined by the curve $\gamma$. We therefore have the following.

Proposition 9.1 For a closed orientable surface $M$, the restriction of the Lie algebra map $\Psi: H_{2 *}^{S^{1}}(L M) \rightarrow \mathcal{O}(\mathcal{M C})$, to the 0 -th equivariant homology $H_{0}^{S^{1}}(L M)$ is the same is the map $\gamma \mapsto f_{[\gamma]}$, from $\mathbb{R} \hat{\pi}$ to the Poisson algebra of functions on the symplectic space $\operatorname{Hom}(\pi, G) / G$, discovered by Goldman in [17].

\section{Appendix A Vector bundles and flat connections}

In this appendix first we review some basic facts relating the space of representations of $\pi_{1}(M)$ the fundamental group of a manifold $M$ in a Lie group $G$ and the space of the isomorphism classes of principal bundles on $M$ equipped with a flat connection. Second we prove some basic facts which at end enables us to show that $\Omega^{*}(M, \operatorname{ad} P)$ is an example of DGLA as it is defined in Section 4. In the end we recall some homology and cohomology with coefficient in a flat bundle. For more details, we refer the reader to Greub, Halperin and Vanstone [18]. Notice that a connection on a principal bundle 
$G \rightarrow P \rightarrow M$ gives rise to a connection on the associated vector bundles. Therefore, we denote the connections on its associated vector bundles with the same notation $\nabla$. The covariant derivative $\nabla: \Omega^{0}(M, E) \rightarrow \Omega^{1}(M, E)$ can be extended by Leibnitz rule to a differential $d_{\nabla}: \Omega^{*}(M, E) \rightarrow \Omega^{*+1}(M, E)$. The flatness of the connection translate into the equation $d_{\nabla}^{2}=0$.

Let $\pi_{1}(M)$ denote the fundamental group of a manifold $M$ with a based point $b$. Let $\operatorname{Hom}\left(\pi_{1}(M), G\right)$ denote the set of all group homomorphisms from $\pi_{1}(M)$ to a Lie group $G$. Let $\mathcal{F}$ denote the set of all pairs $(P, \nabla)$ of principal $G$-bundles $P$ equipped with a flat connection $\nabla$ up to bundle isomorphisms. The universal covering $\pi_{1}(M) \rightarrow \tilde{M} \rightarrow M$ may be viewed as a principal $\pi_{1}(M)$-bundle over $M$ and therefore given a homomorphism $\rho: \pi_{1}(M) \rightarrow G$ one can construct the associated bundle $\tilde{M} \times{ }_{\rho} G$. The canonical connection given by unique lifting property will induce a connection on $\tilde{M} \times{ }_{\rho} G$. This establishes a map $\Phi: \operatorname{Hom}\left(\pi_{1}(M), G\right) \rightarrow \mathcal{F}$ by $\Phi(\rho)=\tilde{M} \times{ }_{\rho} G$. The following statement is the Theorem 6.60 of Morita [23].

Proposition A.1 The map that associates to any flat $G$-bundle over $M$, its holonomy is a one-to-one correspondence between the set of conjugacy classes of flat $G$-bundles and the set of conjugacy classes of homomorphisms $\rho: \pi_{1} \rightarrow G$.

In this paper, we are mainly interested in the adjoint bundle $\mathfrak{g} \rightarrow \operatorname{ad} P \rightarrow M$, and the universal enveloping bundle $U \mathfrak{g} \rightarrow P_{\mathfrak{u}} \rightarrow M$, associated with the adjoint representation Ad: $G \rightarrow \operatorname{Aut}(\mathfrak{g})$ and $\operatorname{Ad}_{u}: G \rightarrow \operatorname{Aut}(U \mathfrak{g})$. Below are a few applications of the previous results.

Corollary A.2 For $x, y \in \Omega^{*}(M, \operatorname{ad} P)$ we have

$$
d_{\nabla}[x, y]=\left[d_{\nabla} x, y\right]+(-1)^{|x|}\left[x, d_{\nabla} y\right] .
$$

Proof The claim may be verified locally. In a local trivialization $U \times G$ of $P$, the connection $\nabla$ is given by a 1 -form $\theta \in \Omega^{1}(M, \mathfrak{g})$. In the corresponding trivialization $U \times \mathfrak{g}$ of ad $P$, the differential $d_{\nabla}$ equals $d+[\theta, \cdot]$. Note that both terms are degree 1 derivations of the bracket.

Recall the definition of the bilinear form $\omega$ from Example 4.2:

$$
\omega: \Omega^{i}(M, \operatorname{ad} P) \times \Omega^{j}(M, \operatorname{ad} P) \stackrel{\wedge}{\rightarrow} \Omega^{i+j}(M, \operatorname{ad} P \otimes \operatorname{ad} P) \stackrel{\langle\cdot, \cdot\rangle}{\longrightarrow} \Omega^{i+j}(M, \mathbb{R}) \stackrel{\int_{M}}{\longrightarrow} \mathbb{R},
$$

when $i+j=2 d$, and zero otherwise.

The next corollary follows from an argument similar to the proof of Corollary A.2 and Stokes' Theorem. 
Corollary A.3 For $x, y \in \Omega^{*}(M, \operatorname{ad} P)$, we have

$$
\omega\left(d_{\nabla} x, y\right)+(-1)^{|x|} \omega\left(x, d_{\nabla} y\right)=0 .
$$

Corollary A.4 For a section $s \in \Gamma\left(P_{\mathfrak{u}}\right)$, we have

$$
d \operatorname{tr}(s)=\operatorname{tr}(\nabla s) \in \Omega^{1}(M, \mathbb{R}) .
$$

Therefore, for $\alpha \in \Omega^{*}\left(M, P_{\mathfrak{u}}\right)$,

$$
d \operatorname{tr}(\alpha)=\operatorname{tr}\left(d_{\nabla} \alpha\right)
$$

Proof Locally $d_{\nabla}=d+[\theta, \cdot]$ and $\operatorname{tr}[\cdot, \cdot]=0$.

Let $E, E_{1}, E_{2}$, and $E_{3}$ denote vector bundles over a compact manifold $M$ of dimension $m$, each endowed with a flat connection $\nabla$. Given a bundle map $\beta: E_{1} \otimes E_{2} \rightarrow E_{3}$, there is a natural wedge product $\wedge: \Omega^{*}\left(M, E_{1}\right) \otimes \Omega^{*}\left(M, E_{2}\right) \rightarrow \Omega^{*}\left(M, E_{3}\right)$. In addition, if $\beta$ is parallel, this map respects the differentials and consequently induces a well-defined cup product $\cup: H^{*}\left(M, E_{1}\right) \otimes H^{*}\left(M, E_{2}\right) \rightarrow H^{*}\left(M, E_{3}\right)$ on the cohomology. A noteworthy special case is the differential graded algebra structure of the differential forms with values in a flat bundle whose fibres are algebras and the parallel transport maps are algebra maps. For instance $\Omega^{*}\left(M, P_{\mathfrak{u}}\right)$ is an associative (not graded commutative) differential graded algebra giving rise to the graded associative algebra $H^{*}\left(M, P_{\mathfrak{u}}\right)$. Let us also consider chains $C_{*}(M, E)$ with values in a bundle $E$. More precisely, $C_{k}(M, E)$ is the vector space generated by pairs $(\sigma, s)$, where $\sigma: \Delta^{k} \rightarrow M$ is a singular chain and $s$ is a flat section of $\sigma^{*} E$. The boundary map $\partial: C_{k}(M, E) \rightarrow$ $C_{k-1}(M, E)$ is defined as $\partial(\sigma, s)=\Sigma_{i=0}^{k}(-1)^{i}\left(\sigma_{i},\left.s\right|_{\sigma_{i}}\right)$, where $\partial \sigma=\Sigma_{i=0}^{k}(-1)^{i} \sigma_{i}$. There is also a cap product $H^{*}\left(M, E_{1}\right) \otimes H_{*}\left(M, E_{2}\right) \rightarrow H_{*}\left(M, E_{3}\right)$. For example Lemma 7.2 uses the Poincaré duality map $\cap[M]: H^{*}(M, \operatorname{ad} P) \rightarrow H_{*}(M, \operatorname{ad} P)$, induced by the bundle map $\beta: \mathbf{R} \otimes \operatorname{ad} P \rightarrow \operatorname{ad} P$, defined by $\beta(r, x)=r x$, where $\mathbb{R} \rightarrow \mathbf{R} \rightarrow M$ is the trivial $\mathbb{R}$-bundle. The Poincaré duality map is an isomorphism [9].

\section{Appendix B Parallel transport and iterated integrals}

Let $\mathbb{R}^{k} \rightarrow E \rightarrow M$ be a vector bundle endowed with a fixed connection $\nabla$ which is not necessarily flat. In this appendix we show that the holonomy of any other a arbitrary connection $\nabla^{\prime}$ (not necessarily flat) along a loop $\gamma$ in $M$ can be expressed by an iterated integral and the holonomy of $\nabla$. This explains the definition of generalized holonomy in Section 5. We presume that this is well known but we do not know a good reference for it. 
To start, we trivialize $E$ over a given path $\gamma:[a, b] \rightarrow M$ and using this trivialization we take $\nabla+\theta \wedge \cdot$ a local expression for $\nabla^{\prime}$. Here, $\theta \in \Omega^{1}\left(M\right.$, End $\left.\left(\mathbb{R}^{k}\right)\right)$ is a matrix valued 1 -form. We aim at finding $t \mapsto P_{t} \in \operatorname{End}\left(\mathbb{R}^{k}\right)$ the parallel transport along $\gamma$. For a vector $v \in \mathbb{R}^{k}, t \mapsto P_{t}(v)$ the parallel transport of $v$ along $\gamma$ is defined by the differential equation,

$$
\nabla_{\dot{\gamma}(t)}^{\prime} P_{t}(v)=0 \text {. }
$$

Since the bundle has been trivialized, we can think of $P_{t}$ as a matrix valued function which satisfies

$$
\nabla_{\dot{\gamma}(t)} P_{t}(v)+\theta(\dot{\gamma}(t)) P_{t}(v)=0 .
$$

Let $\psi_{t}$ be the parallel transport of $\nabla$ and $R_{t} \in \operatorname{End}\left(\mathbb{R}^{k}\right)$ such that $P_{t}=R_{t} \psi_{t}$. Since $\nabla_{\dot{\gamma}(t)} \psi_{t}=0$, by Leibnitz rule the differential equation (B-1) reduces to

$$
\frac{d R_{t}}{d t}+\theta(\dot{\gamma}(t)) R_{t}=0
$$

in the matrix valued functions for the initial value $R_{0}=$ id. Let $A(t)=\theta(\dot{\gamma}(t))$, the first observation is that $(\mathrm{B}-2)$ is equivalent to

$$
R_{t}=\mathrm{id}+\int_{0}^{t} A(s) R_{s} d s
$$

thus $R_{t}$ is the fixed point of the operator $\phi \mapsto T(\phi)$, where

$$
T(\phi)(t)=\mathrm{id}+\int_{0}^{t} A(s) \phi(s) d s .
$$

Let $b \in[0,1]$ be such that $R=\int_{0}^{1}\|A(s)\| d s<1$, then

$$
\left\|T\left(\phi_{1}\right)-T\left(\phi_{2}\right)\right\| \leq R\left\|\phi_{1}-\phi_{2}\right\|,
$$

hence $T$ is a contracting operator on the Banach space of continuous matrix valued functions on $[0, b]$. It is a known fact that such operators have unique fixed point. In fact the fixed is the limit point of the Cauchy sequence $\phi, T(\phi), T^{2}(\phi), \cdots$ where $\phi$ is arbitrary.

By some classical techniques we can show that this solution can be extended to the entire interval $[0,1]$ in order to obtain a fixed point for the operator $T$ on the Banach space of continuous matrix valued function on $[0,1]$.

Proposition B.1 Equation (B-1) with given initial value has a unique solution which is differentiable and satisfies $(\mathrm{B}-3)$. 
Applying (B-3) repeatedly we obtain

$$
\begin{aligned}
R_{1}=\mathrm{id} & +\int_{0}^{1} A\left(t_{1}\right) R_{t_{1}} d t_{1} \\
=\mathrm{id} & +\int_{0}^{1} A\left(t_{1}\right) d t_{1}+\int_{0}^{1} \int_{0}^{t_{1}} A\left(t_{1}\right) A\left(t_{2}\right) R_{t_{2}} d t_{2} d t_{1} \\
\vdots & \mathrm{id}+\int_{0}^{1} A\left(t_{1}\right) d t_{1}+\int_{0}^{1} \int_{0}^{t_{1}} A\left(t_{1}\right) A\left(t_{2}\right) d t_{2} d t_{1} \\
& +\int_{0}^{1} \int_{0}^{t_{1}} \int_{0}^{t_{2}} A\left(t_{1}\right) A\left(t_{2}\right) A\left(t_{3}\right) d t_{3} d t_{2} d t_{1}+\cdots \\
& +\int_{0}^{1} \int_{0}^{t_{1}} \cdots \int_{0}^{t_{n}} A\left(t_{1}\right) A\left(t_{2}\right) \cdots A\left(t_{n}\right) d t_{n} \cdots d t_{2} d t_{1}+\cdots
\end{aligned}
$$

Note that since $A\left(t_{i}\right)=i_{\frac{\partial}{\partial t_{i}}} \mathrm{ev}_{i}^{*}(\theta)$, we have

$$
\int_{0}^{1} \int_{0}^{t_{1}} \cdots \int_{0}^{t_{n}} A\left(t_{1}\right) A\left(t_{2}\right) \cdots A\left(t_{n}\right) d t_{n} \cdots d t_{2} d t_{1}=\int_{\gamma} \int_{\Delta^{n}} \operatorname{ev}_{1}^{*}(\theta) \operatorname{ev}_{2}^{*}(\theta) \cdots \operatorname{ev}_{n}^{*}(\theta) .
$$

Hence, $\quad R_{1}=\psi_{1}+\int_{\gamma} \int_{\Delta^{1}} \operatorname{ev}_{1}^{*}(\theta) \psi_{1}+\int_{\gamma} \int_{\Delta^{2}} \operatorname{ev}_{1}^{*}(\theta) \operatorname{ev}_{2}^{*}(\theta) \psi_{1}$

$$
+\cdots+\int_{\gamma} \int_{\Delta^{n}} \operatorname{ev}_{1}^{*}(\theta) \operatorname{ev}_{2}^{*}(\theta) \cdots \operatorname{ev}_{n}^{*}(\theta) \psi_{1}+\cdots
$$

Remark B.2 This explains the formula for the generalized holonomy in Section 6 and confirms the convergence of the generalized holonomy. To sum up, the solution of a general time-dependent system of linear equations $\dot{X}=A(t) X$ with initial condition $X(0)=X_{0}$ may be expressed in terms of a power series each of whose terms is a Chen iterated integral. Note that in dealing with a time-independent system, in which $A(t)=A$, for all $t \in \mathbb{R}$, this formula reduces to $X(t)=e^{t A} X_{0}$, since the volume of the $n$-simplex is $\frac{1}{n !}$.

\section{References}

[1] MF Atiyah, R Bott, The moment map and equivariant cohomology, Topology 23 (1984) 1-28 MR721448

[2] J-L Brylinski, Loop spaces, characteristic classes and geometric quantization, Progress in Mathematics 107, Birkhäuser, Boston (1993) MR1197353 
[3] A S Cattaneo, J Fröhlich, B Pedrini, Topological field theory interpretation of string topology, Comm. Math. Phys. 240 (2003) 397-421 MR2005849

[4] A S Cattaneo, C A Rossi, Higher-dimensional BF theories in the Batalin-Vilkovisky formalism: the BV action and generalized Wilson loops, Comm. Math. Phys. 221 (2001) 591-657 MR1852055

[5] M Chas, D Sullivan, String topology, to appear in Ann. of Math.

[6] M Chas, D Sullivan, Closed string operators in topology leading to Lie bialgebras and higher string algebra, from: "The legacy of Niels Henrik Abel", Springer, Berlin (2004) 771-784 MR2077595

[7] K-t Chen, Iterated integrals of differential forms and loop space homology, Ann. of Math. (2) 97 (1973) 217-246 MR0380859

[8] K T Chen, Iterated path integrals, Bull. Amer. Math. Soc. 83 (1977) 831-879 MR0454968

[9] J M Cohen, Poincaré 2-complexes II, Chinese J. Math. 6 (1978) 25-44 MR0488020

[10] R L Cohen, J D S Jones, A homotopy theoretic realization of string topology, Math. Ann. 324 (2002) 773-798 MR1942249

[11] R Cohen, J Klein, D Sullivan, The homotopy invariance of the string topology loop product and string bracket arXiv:math.GT/0509667

[12] R L Cohen, A A Voronov, Notes on string topology, from: "String topology and cyclic homology”, Adv. Courses Math. CRM Barcelona, Birkhäuser, Basel (2006) 1-95 MR2240287

[13] W L Gan, V Ginzburg, Hamiltonian reduction and Maurer-Cartan equations, Mosc. Math. J. 4 (2004) 719-727, 784 MR2119146

[14] E Getzler, J D S Jones, S Petrack, Differential forms on loop spaces and the cyclic bar complex, Topology 30 (1991) 339-371 MR1113683

[15] R Godement, Topologie algébrique et théorie des faisceaux, Actualités Sci. Ind. No. 1252. Publ. Math. Univ. Strasbourg. No. 13, Hermann, Paris (1958) MR0102797

[16] W M Goldman, The symplectic nature of fundamental groups of surfaces, Adv. in Math. 54 (1984) 200-225 MR762512

[17] W M Goldman, Invariant functions on Lie groups and Hamiltonian flows of surface group representations, Invent. Math. 85 (1986) 263-302 MR846929

[18] W Greub, S Halperin, R Vanstone, Connections, curvature, and cohomology. Vol. II: Lie groups, principal bundles, and characteristic classes, Pure and Applied Mathematics 47-II, Academic Press (1973) MR0336651

[19] V W Guillemin, S Sternberg, Supersymmetry and equivariant de Rham theory, Mathematics Past and Present, Springer, Berlin (1999) MR1689252 
[20] R S Hamilton, The inverse function theorem of Nash and Moser, Bull. Amer. Math. Soc. (N.S.) 7 (1982) 65-222 MR656198

[21] Y Karshon, An algebraic proof for the symplectic structure of moduli space, Proc. Amer. Math. Soc. 116 (1992) 591-605 MR1112494

[22] S A Merkulov, De Rham model for string topology, Int. Math. Res. Not. (2004) 29552981 MR2099178

[23] S Morita, Geometry of differential forms, Translations of Mathematical Monographs 201, American Mathematical Society, Providence, RI (2001) MR1851352

[24] A Schwarz, A-model and generalized Chern-Simons theory, Phys. Lett. B 620 (2005) 180-186 MR2149784

Max-Planck Institut für Mathematik

Vivatsgasse 7, Bonn 53111, Germany

Long Island University, CW Post College

Brookville NY 11548, USA

abbaspou@mpim-bonn.mpg.de, mzeinalian@liu.edu

http://guests.mpim-bonn.mpg.de/abbaspou/

Received: 15 January 2007 